\title{
Minimal Interference Beam Size/Profile Measurement Techniques Applicable to the Collider
}

W. Nexsen, S. Dutt, S. Kauffmann, V. Lebedev, A. Maschke,

N. Mokhov, R. Richardson, E. Tsyganov, and A. Zinchenko

\author{
Superconducting Super Collider Laboratory* \\ 2550 Beckleymeade Ave. \\ Dallas, TX 75237
}

May 1993

"Operated by the Universities Research Association, Inc., for the U.S. Department of Energy under Contract No. DE-AC35-89ER40486. 


\title{
Minimal Interference Beam Size/Profile Measurement Techniques Applicable to the Collider
}

W. Nexsen, S. Dutt, S. Kauffmann, V. Lebedev, A. Maschke,

N. Mokhov, R. Richardson, E. Tsyganov, and A. Zinchenko

\begin{abstract}
The imaging of synchrotron radiation (SR) has been suggested as a techrique for providing a continuous, non-interfering monitor of the beam profile in the Collider rings at the Superconducting Super Collider. A closer examination has raised questions concerning the applicability of SR imaging in this case because of the diffraction broadening of the image, the requirements for axial space and location in the lattice, and the complexity of the system. We have surveyed the known, alternative, minimal interference techniques for measuring beam size and have evaluated them for possible Collider usage. We conclude that of the approaches that appear feasible, all require at least some development for our usage and that the development of an electron beam probe offers the rest promise. We recommend that flying wires be used for cross-checking and calibrating the electron beam probe diagnostic and for luminosity measurements when the highest accuracy is required, but flying wires should not be used as the primary diagnostic because of their limited lifetime.
\end{abstract}




\subsection{INTRODUCTION}

The Superconducting Super Collider (SSC) is designed to produce and maintain a beam collisional luminosity at the interaction points greater than $10^{33} \mathrm{~cm}^{-2} \mathrm{~s}^{-1}$ at $20 \mathrm{TeV} / \mathrm{c}$. The baseline parametric requirements necessary to obtain this luminosity include an rms beam spot radius, $\sigma$, of $5 \mu \mathrm{m}$ at the interaction point. The concept of normalized emittance is used to relate this value to measurements made earlier in the injector chain and at other places around the Collider. For monoenergetic beams following a stable closed orbit, the beam sizes in each of the transverse planes and at any two locations are related by

$$
\left(\gamma \sigma^{2} / \beta_{\mathrm{H}, \mathrm{V}}\right)_{1}=\left(\gamma \sigma^{2} / \beta_{\mathrm{H}, \mathrm{V}}\right)_{2}=\varepsilon_{\mathrm{N}}
$$

where $\beta_{H, V}$ is the horizontal or vertical lattice beta-function value at the point of measurement, $\gamma$ is the relativistic parameter, and $\varepsilon_{N}$ is the normalized emittance. The Collider luminosity goals require a final baseline normalized emittance of $1 \pi \mathrm{mm}$-mrad. Since the emittance is expected to grow somewhat through the injector chain, this is the maximum value that should be measured. Beam size/profile measurements will be an important tool for detecting and controlling emittance growth. Table 1 lists the expected beam size for a $20 \mathrm{TeV} / \mathrm{c}$ beam with this baseline emittance.

TABLE 1. COLLIDER BASELINE BEAM SIZE.

\begin{tabular}{|l|l|l|}
\hline \multicolumn{1}{|c|}{ Location } & $\beta_{\max } / \beta_{\min }$ & \multicolumn{1}{|c|}{$\sigma_{\max } / \sigma_{\min }$} \\
\hline Interaction point & $0.5 \mathrm{~m}$ & $5 \mu \mathrm{m}$ \\
Secondary focus & $60 \mathrm{~m} / 30 \mathrm{~m}$ & $55 \mu \mathrm{m} / 39 \mu \mathrm{m}$ \\
Utility straight & $355 \mathrm{~m} / 46 \mathrm{~m}$ & $129 \mu \mathrm{m} / 46 \mu \mathrm{m}$ \\
Utility dogleg & $600 \mathrm{~m} / 400 \mathrm{~m}$ & $173 \mu \mathrm{m} / 141 \mu \mathrm{m}$ \\
\hline
\end{tabular}

Since the beam is never monoenergetic, the measured beam size will be larger if there is dispersion in the plane of measurement, i.e.,

$$
\sigma_{\text {meas }}=\left(\sigma_{\varepsilon}^{2}+\sigma_{x}^{2}\right)^{1 / 2}
$$

where $\sigma_{\varepsilon}=\left(\beta_{x} \varepsilon_{N} / \gamma\right)^{1 / 2}$ and $\sigma_{x}=D \sigma_{p} / p$. For dispersion to produce a $<5 \%$ increase in the measured beam size it is necessary that $\sigma_{x}<\sigma_{\varepsilon} / 3$. The baseline value of the momentum spread is $5 \times 10^{-5}$; residual dispersion of $\sim 80 \mathrm{~cm}$ in the utility straight or $\sim 120 \mathrm{~cm}$ in the utility dogleg would be necessary to produce a $5 \%$ error. The dispersion in these regions is expected to be much less than this value; consequently, profile measurements made here should not be appreciably perturbed by it.

For most of the techniques we have surveyed, measurements where the beam is largest, i.e., in the utility dogleg, will be the most accurate; unless otherwise noted in our assessments, we will assume that the measurement will be made there. An absolute measurement of beam radial size, $\sigma_{\mathrm{H}, \mathrm{V}}$, with an error $<5 \%$ is desirable. This allows a determination of absolute luminosity good to approximately $\pm 10 \%$ if the errors in all the other factors involved in calculating luminosity are kept small. Changes the size of $\sim 1 \%$ should be resolvable in order to observe emittance growth in a time period short compared with the emittance lifetime.

The first requirement for any beam size diagnostic for a cyclic accelerator is that it have minimal effect on the beam. This precludes the use of flags or foils and multiwire grids. (Grids may be useful in the transfer lines.) The flying wire technique, described below, can yield an accurate measurement of beam line-density profile but is not a continuous measurement. The large accelerators, for which the flying wire is the main profile diagnostic, have expended a great deal of money and effort to develop and build additional diagnostics to provide a continuous, non-interfering radial profile monitor. Examples include the attempt to develop a turn-by-turn beam profile monitor for the Fermilab booster, which should be capable of extracting profiles every 1.6-2.7 $\mu \mathrm{s},{ }^{1}$ and the imaging of synchrotron radiation (SR) on the Tevatron and planned for the Large 
Hadron Collider (LHC). The Collider baseline requirements recognize the need for such a diagnostic, recommending the use of SR imaging in addition to flying wires. ${ }^{2}$ In the remainder of this document we first examine the limitations of the flying wire that drive the need for an additional method of profile measurement, point out the problems faced in using SR imaging on the Collider, and describe the electron beam probe that we believe offers the best alternative beam size diagnostic. In the Appendix we describe the remainder of the known techniques that we have surveyed.

\subsection{BEAM SIZE DIAGNOSTICS}

\subsection{Flying Wire Scanners}

At present the primary profile diagnostic for hadron synchrotrons is the flying wire scanner. ${ }^{3-10}$ This diagnostic, pictorially shown in Figure 1 (from Reference 4), employs a thin fiber or wire that is swept through the beam while detectors downstream record the radiation induced by beam interaction as a function of wire position. The scanning system is programmed to give the wire a trapezoidal velocity profile, accelerating the wire to the desired constant velocity at which it passes through the beam, and stopping the wire after one transit or "flight." For the Collider the minimum scanning velocity is limited by the allowed temperature rise of the wire. For a carbon filament bombarded by $2-20 \mathrm{TeV}$ protons, the temperature rise during one pass through the beam is of the order of

$$
\Delta T=20 I /\left(\sigma_{\min } v\right) \quad\left({ }^{\circ} \mathrm{C}\right),
$$

where $I$ is the average beam current, $v$ is the wire velocity $(\mathrm{m} / \mathrm{s})$ normal to the beam, and $\sigma_{\min }(\mathrm{m})$ is the rms beam radius along the minor axis, assuming the scan is taken in the direction of the major axis of the beam to minimize errors. A maximum temperature rise per pass of the order of $3200^{\circ} \mathrm{C}$ may be allowable as long as the temperature cools to ambient prior to the next pass. For baseline current and $\sigma_{\min }=141 \mu \mathrm{m}$, a velocity greater than $3.2 \mathrm{~m} / \mathrm{s}$ normal to the beam is necessary to keep the single pass temperature rise below the limit. Since the geometry is such that the wire cuts through the beam at an angle, the required rotational speed of the wire is of the order of $5 \mathrm{~m} / \mathrm{s}$. It would be preferable to use a greater wire speed both to reduce the maximum temperature rise and to reduce the emittance growth and nuclear reactions per scan. Fermi National Accelerator Laboratory uses systems with speeds of $\sim 5 \mathrm{~m} / \mathrm{s}$, while CERN has developed a monitor whose $36-\mu \mathrm{m}$ filament has a velocity normal to the beam of $20 \mathrm{~m} / \mathrm{s}$ ( $30 \mathrm{~m} / \mathrm{s}$ rotational speed).

Broadening of the measured profile due to the finite size of the fiber is small. A $36-\mu \mathrm{m}$ diameter fiber has an effective rms radius of $9 \mu \mathrm{m}$, which, when added in quadrature with $\sigma_{\max }$ at the utility dogleg, gives a little more than $0.2 \%$ broadening. While $1-\mu \mathrm{m}$ resolution has been reported for wires moving at much slower speeds, no numbers are available for higher speeds. Similar resolution should be possible, however, since the main error will be due to uncertainty in the wire position, and the time of passage of the wire through the beam is so short $(-22 \mu \mathrm{s})$ that vibrational motion of the wire during that time probably can be ignored.

The interaction between beam and wire can produce beam emittance growth and a radiation load on nearby magnets, but neither of these appears to be serious in the Collider if the wire is flown intermittently. The emittance growth from one pass of the wire through the beam can be estimated as follows: the rms scatter angle is given by $\theta=\left(p_{0} / p\right)\left(L / L_{\mathrm{T}}\right)^{1 / 2}$, where $p_{0}=14 \mathrm{MeV} / \mathrm{c}, p$ is the beam momentum, and $L_{\mathrm{T}}$ the radiation length of the material $\left(0.27 \mathrm{~m}\right.$ for carbon). The effective thickness of the fiber is $L=\left(\pi f d^{2}\right) /(4 \mathrm{v})$, where $f$ is the frequency of particle rotation, $d$ is the fiber diameter $(\mathrm{m})$, and $v$ is the wire velocity transverse to the beam $(\mathrm{m} / \mathrm{s})$. The growth of the normalized emittance from Coulomb scattering is $\Delta \varepsilon_{n}=\gamma \beta \theta^{2} / 2$, where $\beta$ is the lattice beta-function. Table 2 gives the estimated emittance increase per scan of a 36- $\mu \mathrm{m}$-diameter carbon fiber with a scanning velocity of $20 \mathrm{~m} / \mathrm{s}$ at the utility dogleg where $\beta_{\max } \approx 600 \mathrm{~m}$. 


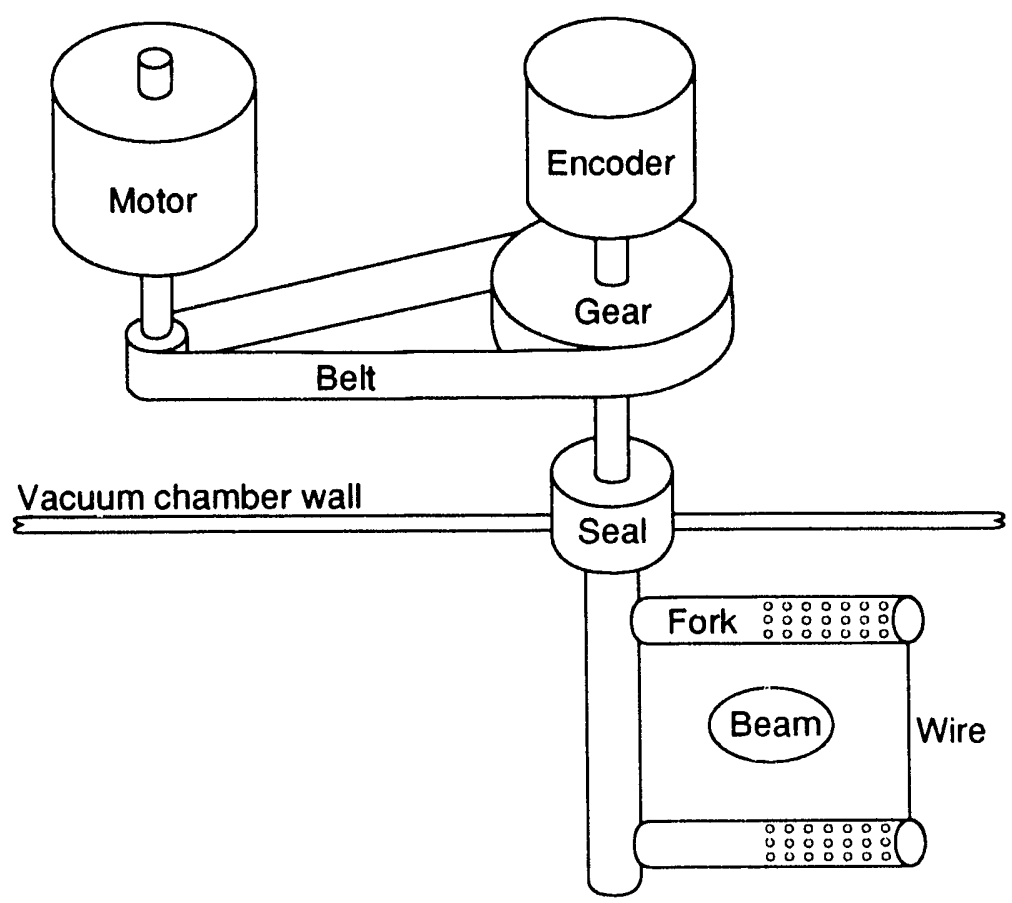

TIP-04602

Figure 1. Pictorial Drawing of Wire Assembly (From Reference 2).

TABLE 2. EMITTANCE INCREASE/SCAN IN COLLIDER.

\begin{tabular}{|c|c|c|c|c|c|}
\hline$p($ ToV/c) & $\gamma$ & $f(\mathbf{k H z})$ & $\Delta \varepsilon_{n}(\mathbf{m})$ & $\varepsilon_{n}(\mathbf{m})$ & $\Delta \varepsilon_{n} / \varepsilon_{n}$ \\
\hline 2 & 2132 & 3.44 & $2 \mathrm{E}(-11)$ & $1 \mathrm{E}(-6)$ & $2 \mathrm{E}(-5)$ \\
20 & 21320 & 3.44 & $2 \mathrm{E}(-12)$ & $1 \mathrm{E}(-6)$ & $2 \mathrm{E}(-6)$ \\
\hline
\end{tabular}

Obviously the emittance growth per pass will not be the limiting factor in the use of the flying wire in the Collider as long as it is flown only intermittently. Similar calculations show a problem, however, if the same diagnostic were used in the Low Energy Booster (LEB). This is shown in Table 3, where we have taken $\beta_{\max } \approx 19 \mathrm{~m}$.

TABLE 3. EMITTANCE INCREASE/SCAN IN LEB.

\begin{tabular}{|c|c|c|c|c|c|}
\hline$p(\mathrm{GeV} / \mathrm{c})$ & $\gamma$ & $f(\mathrm{MHz})$ & $\Delta \varepsilon_{\mathrm{n}}(\mathrm{m})$ & $\varepsilon_{\mathrm{n}}(\mathrm{m})$ & $\Delta \varepsilon_{\mathrm{n}} / \varepsilon_{\mathrm{n}}$ \\
\hline 1.22 & 1.64 & 0.44 & $1.7 \mathrm{E}(-7)$ & $6 \mathrm{E}(-7)$ & 0.28 \\
12 & 12.8 & 0.56 & $1.8 \mathrm{E}(-8)$ & $6 \mathrm{E}(\mathrm{w})$ & 0.03 \\
\hline
\end{tabular}

A thinner wire might be used in the LEB, but experience has shown that this may not be compatible with the high velocity, and reliability, already a problem, would probably suffer.

For $72 \mathrm{~mA}$ of $20-\mathrm{TeV}$ protons, the estimated number of nuclear interactions induced per scan of a $36-\mu \mathrm{m}$-diameter carbon fiber moving at $20 \mathrm{~m} / \mathrm{s}$ is of the order of $7 \times 10^{7}$. To put this value in perspective, the expected loss to the scrapers during steady operation is a maximum of $4 \times 10^{8} \mathrm{p} / \mathrm{s}$.

For each flight of the CERN scanner the wire is subjected to an acceleration greater than $100 \mathrm{~g}$, mechanical failure is common, and the wire has an average life of only a few thousand flights. For present designs a broken wire means the diagnostic is lost until the run is stopped. While a slower scanning speed may result in a 
larger number of flights between failures, it is likely that the mean time between failures will be such that flights will have to be limited to an average rate of the order of a few per hour to guarantee availability throughout a Collider run. Options to increase this rate would involve redundancy, the development of remote wire replacement systems, and extending the wire lifetime. It is probably not practicable to consider using redundancy to increase the availability by a large amount, considering the cost and space required. The capability to change a broken wire remotely is not available at present and would require development. Since the wire mounting systems are in a rather mature stage of development, a large gain in wire lifetime is not to be expected. A suggestion to allow multirotations of the wire to avoid the accelerative forces is not an option. High acceleration would still be required in starting and stopping the wire in order to avoid passage through the beam at slow speeds unless we propose to run it continuously. In this case, if we assume a rotational speed of $30 \mathrm{~m} / \mathrm{s}$ of the wire at the end of a $0.2-\mathrm{m}$ fork, the rotational frequency will be $24 \mathrm{~Hz}$ and the beam will be cut at a rate of $48 \mathrm{~Hz}$. At this rate the wire temperature will rapidly ratchet up to the failure value since it will not have time to cool completely between passages. Even if it avoided this fate, the nuclear interaction rate will be almost a factor of ten greater than that due to the steady state scrapers, and emittance growth will amount to $\sim 35 \% / \mathrm{h}$.

We believe that flying wire scanners will be needed in the Collider because of their ability to measure profiles with high accuracy. They should not be used as the primary beam size diagnostic because of their limited time between failures, but should be used for cross-checking and calibrating other measurements and when precise measurements are needed to determine luminosity.

\subsection{Synchrotron Radiation Imaging}

The imaging of synchrotron radiation emitted by an accelerated charge has become a standard diagnostic for electron synchrotrons, but at present only a few proton accelerators ${ }^{11-14}$ operate at an energy where the radiation is detectable. The prospects are good for SR imaging on these accelerators, but diffraction broadening of the beam image will be a serious problem for the Collider, where the beam radius will be much smaller.

In designing a SR profile monitor for the Collider ring, we must consider the two sources of light emission that yield different spectral and angular characteristics. These are the radiation emitted during passage of ultrarelativistic protons through (1) a normal bending dipole, or (2) the edge of a dipole field, a wiggler, or a short magnet. ${ }^{15-19}$ An ultrarelativistic $(\gamma>>1)$ charged particle interacting with a transverse magnetic field emits radiation that is mostly confined in a cone of half angle $\sim 1 / \gamma$ whose axis is tangent to the particle trajectory. The observer in Figure 2 would see a pulse of light emanating from an arc of length $\sim 2 \mathrm{~g} / \gamma$, provided the dipole is at least this long. This is case (1) above. The time structure of the pulse received by the observer can be estimated from the difference between the particle's time of flight across the arc and the time for a photon to traverse the chord of the arc:

$$
\Delta t=(2 \mathrm{Q} / \beta \gamma \mathrm{c})(1-\beta) \approx 4 \mathrm{Q} / 3 \mathrm{c} \gamma^{3} .
$$

Assuming a Gaussian shape to the pulse with $\sigma_{t} \approx \Delta t / 2$, the Fourier transform of the pulse gives a Gaussian frequency spectrum with critical frequency defined by $\omega_{c}=\sigma_{f}=3 c \gamma^{3} / 2 \mathrm{~g}$. The typical vertical half opening angle of the SR amplitude for $\omega<<\omega_{c}$ is approximately ${ }^{18}$

$$
\alpha_{\mathrm{c}} \approx(1 / \gamma)\left(\omega_{\mathrm{c}} / \omega\right)^{1 / 3}
$$

On the other hand, for case (2) above, at the dipole edge where the field changes with a characteristic length $L<2 \mathrm{Q} / \gamma(\alpha<<1 / \gamma$ in Figure 2), the fall- or rise-time of the radiation from the same ultrarelativistic particle leaving or entering the field will be

$$
\tau \approx(L / \mathrm{c})(1-\beta) \approx L / 2 \mathrm{c} \gamma^{2},
$$




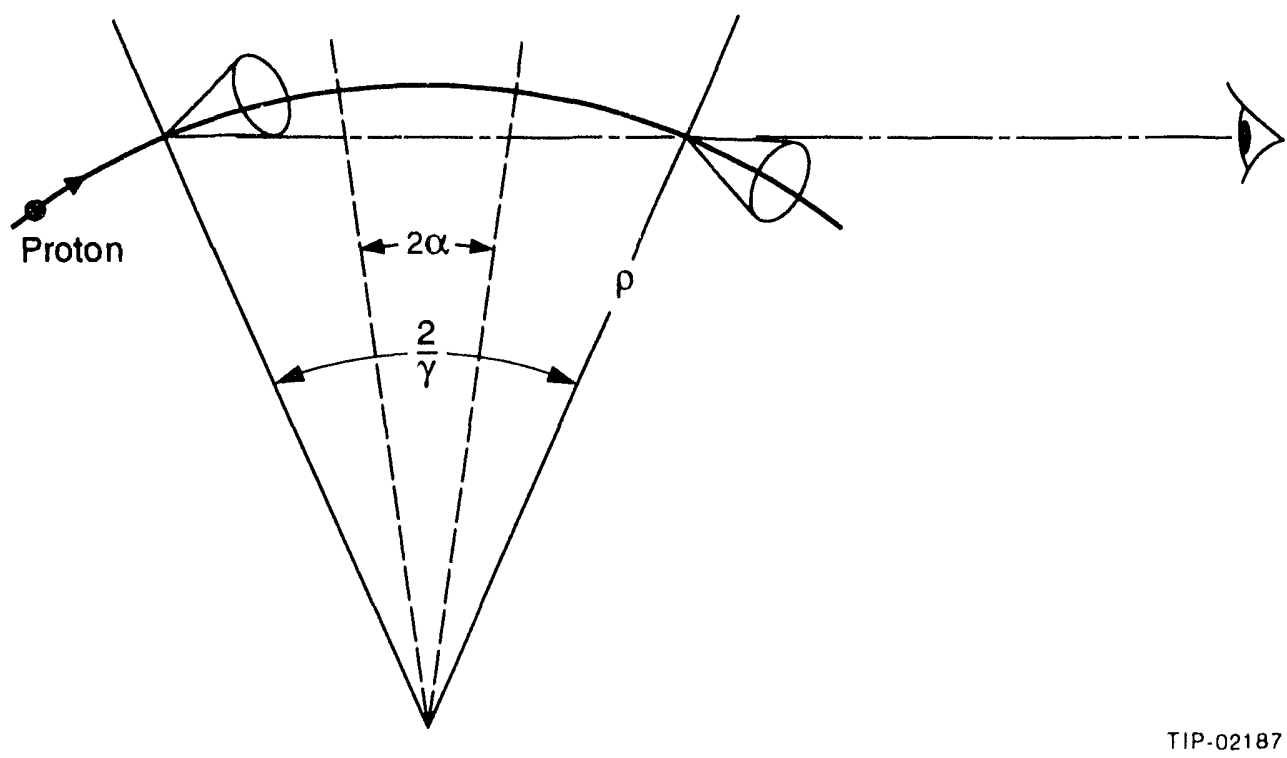

Figure 2. Arc Visible to SR Observer.

and the spectral bandwidth will extend out to $\omega_{\mathrm{s}} \approx 2 \pi / \tau \approx 4 \pi \mathrm{c} \gamma^{2} / L$. The ratio of $\omega_{\mathrm{s}}$ to $\omega_{\mathrm{c}}$ is

$$
\omega_{\mathrm{s}} / \omega_{\mathrm{c}}=8 \pi / 3 \alpha \gamma \gg 1 \text {. }
$$

This ability of a magnetic field discontinuity to shift the SR spectrum to shorter wavelengths was first demonstrated experimentally on the CERN Super Proton Synchrotron (SPS). ${ }^{11}$

A further useful property of the edge radiation is that the opening angle of the radiation cone is greatly increased for $\lambda \gg>\lambda_{s}$. This characteristic angle is

$$
\alpha_{\mathrm{s}} \approx(2 \lambda / L)^{1 / 2} \approx(1 / \gamma)\left(\lambda / \lambda_{\mathrm{s}}\right)^{1 / 2}=(1 / \gamma)\left(\omega_{\mathrm{s}} / \omega\right)^{1 / 2}
$$

Note that the angle is independent of beam energy as long as the condition $\lambda \gg \lambda_{s}$ is satisfied.

The CERN group has designed its measurement to work in the near $u$ aviolet $(\lambda \sim 200 \mathrm{~nm})$, near the short wavelength boundary of the air transmission window. In our assessmer. ve assume that any Collider system will also work in this wavelength region. Some reduction in tiffraction would be obtained by working at shorter wavelength, but at the expense of having the entire optical path in vacuum and possibly having to use a special reflecting optics design. For imaging in the visible to be possible over the whole Collider energy range, it will be necessary to work with the radiation from the edge field of a dipole. (A separate dedicated short magnet would be of little value since a dipole field would still be required to bend the beam out of the path of the SR.) While at $20 \mathrm{TeV}$ in the Collider the critical wavelength, $2 \pi \mathrm{c} / \omega_{\mathrm{c}}$, of the normal dipole SR is $\sim 5 \mathrm{~nm}$, at $2 \mathrm{TeV}$ it is a thousand times longer, in the infrared region of the spectra, and the power emitted will be practically nonexistent in the visible and below. For the edge field, however, if we take $L \approx 0.1 \mathrm{~m}$, then at $20 \mathrm{TeV}, \lambda_{\mathrm{s}}=c \tau \approx 0.1 \mathrm{~nm}$, while at $2 \mathrm{TeV}, \lambda_{\mathrm{s}} \approx 10 \mathrm{~nm}$.

Because of the large Collider radius of curvature, a rather long drift space will be required to separate the SR from the beam. This means that the source for our imaging must be the upstream edge of an isolated dipole or the last dipole in a string. A portion of the separation drift space will be through the magnet or magnets and end spool, but an additional length of unobstructed, enlarged warm beam tube will also be required to ensure that the SR extraction mirror does not limit the beam aperture. We further require that the beam be large and dispersion be small at the source. Two possible locations in each ring have been identified that meet these 
criteria: the entrance edge of the last dipole in the dispersion suppressor at the exit from the arc into the west utility straight section, and the entrance edge of the upstream dogleg magnet in the west utility straight section. The more promising location of these is the dogleg bending magnet because the beam size is the largest (outside of the IR region), dispersion is small, and access is relatively good. A preliminary layout (Figure 3) suggests that it should be possible to view the entrance edge of the dipole with an extraction mirror located $27 \mathrm{~m}$ from the edge. A mirror located there with $20 \mathrm{~mm} \times 20 \mathrm{~mm}$ projected area centered on the exiting light pattern would not intrude into the beam tube aperture. Because of the wide opening angle of the edge radiation, the mirror will be filled by the radiation and will act as a limiting aperture, determining the size of the diffraction pattern from a point source. If we assume that the radiation is uniform across the mirror to estimate the minimum size of the diffraction pattern, that the focusing element is located close to the mirror, and that the system has a 1:1 magnification, then we estimate a rms radius $\sigma_{\mathrm{d}} \approx 120 \mu \mathrm{m}$ for the intensity of the diffraction pattern of a point source. The effect of this diffraction broadening is given in Table 4 . From Table 4 we see that the worst broadening at this location is of the order of $30 \%$.

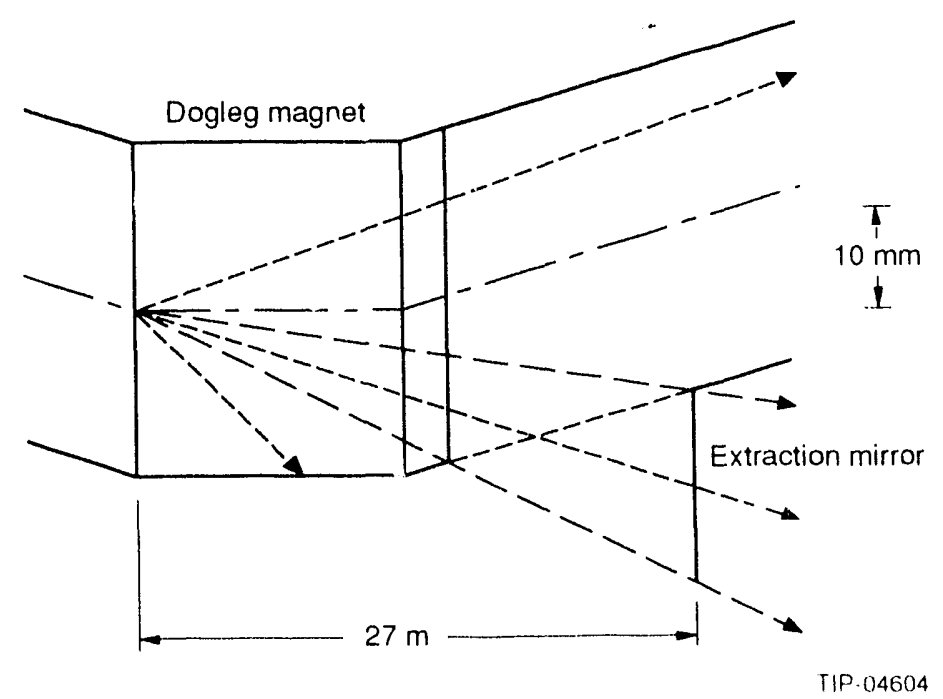

Figure 3. Extractlon of Edge SR from Dogleg Magnet.

TABLE 4. DIFFRACTION BROADENING OF COLLIDER EDGE FIEID SR IMAGE.

\begin{tabular}{|c|c|c|c|c|c|c|}
\hline $\begin{array}{c}E \\
(T \odot V)\end{array}$ & $\begin{array}{c}\lambda \\
(\mathbf{n m})\end{array}$ & $\begin{array}{c}\sigma_{d} \\
(\mu \mathrm{m})\end{array}$ & $\begin{array}{c}\sigma_{\mathbf{H}} \\
(\mu \mathrm{m})\end{array}$ & $\begin{array}{c}\sigma_{H I} \\
(\mu \mathrm{m})\end{array}$ & $\begin{array}{c}\sigma_{\mathbf{V}} \\
(\mu \mathrm{m})\end{array}$ & $\begin{array}{c}\sigma_{\mathbf{V}} \\
(\mu \mathrm{m})\end{array}$ \\
\hline 2 & 200 & 120 & 547 & 560 & 446 & 462 \\
20 & 200 & 120 & 173 & 211 & 141 & 185 \\
\hline
\end{tabular}

$\beta_{\mathrm{H}}=600 \mathrm{~m}, \beta_{\mathrm{V}}=400 \mathrm{~m}$

$\sigma_{\mathrm{HI}, \mathrm{VI}}=\left(\sigma_{\mathrm{H}, \mathrm{V}^{2}}+\sigma_{\mathrm{d}}^{2}\right)^{1 / 2}$

For beam energies below approximately $6 \mathrm{TeV}$, only the edge radiation will contribute to the $200-\mathrm{nm}$ signal; above that energy the critical wavelength for the normal dipole radiation will be shorter than $200 \mathrm{~nm}$ and will contribute to the image. This contribution will be much brighter than that due to the edge radiation because of the longer source length and higher average field. In the horizontal direction, the extraction mirror will remain almost completely filled because of the sweeping of the beam, and it will determine the size of the horizontal beam image, leaving it practically unchanged from the edge field value. However in the vertical direction the light cone angle shrinks, the extraction mirror is not filled, and the diffraction broadening is greatly increased. Adopting the nomenclature of Reference 20, the vertical diffraction broadening in the 
dipole field is estimated in Table 5. The vertical broadening has grown to about $76 \%$ at full beam energy because of the contribution of the much brighter normal dipole radiation.

TABLE 5. VERTICAL BROADENING OF DIPOLE FIELD SR IMAGE.

\begin{tabular}{|c|c|c|c|c|c|}
\hline $\begin{array}{c}E \\
(\mathrm{TeV})\end{array}$ & $\begin{array}{c}\lambda \\
(\mathbf{n m})\end{array}$ & $\begin{array}{c}\sigma_{\mathrm{V}} \\
(\mu \mathrm{m})\end{array}$ & $\begin{array}{c}\sigma_{\psi} \\
(\mu \mathrm{rad})\end{array}$ & $\begin{array}{c}\sigma_{\mathrm{dV}} \\
(\mu \mathrm{m})\end{array}$ & $\begin{array}{c}\sigma_{\mathrm{V}} \\
(\mu \mathrm{m})\end{array}$ \\
\hline 20 & 200 & 141 & 111 & 204 & 248 \\
\hline
\end{tabular}

$$
\begin{aligned}
& \sigma_{\Psi}=0.756(\lambda / 2 \pi \varrho)^{1 / 3} \\
& \sigma_{d V}=0.113 \lambda \sigma_{\psi}
\end{aligned}
$$

By comparing SR images with profiles obtained from flying wire measurements it should be possible to unfold the $\sigma_{\mathrm{dH}, \mathrm{V}}$ due to diffraction broadening and to incorporate these values into algorithms for correcting the images. If we are mainly interested in looking for relative changes in the size of the beam, this may prove satisfactory; however, to meet the requirement that changes in the radius $\sigma_{V}$ of the order of $1 \%$ be resolvable means that changes of $\sigma_{\mathrm{VI}}$ of the order $1 / 3 \%$ must be resolvable. The diagnostic would occupy a lot of beamline space and would have a rather elaborate optical train to keep in alignment. Figure 4 shows the system used at the Large Electron-Positron Collider (LEP) at CERN. Diffraction could be reduced by imaging at shorter wavelength, but this would require that the optical train and detector be in the beam vacuum. Such a system would require a research and development effort. Still it could only be used in the Collider and High Energy Booster (HEB) because of the strong dependence of SR on energy. We believe that effort might better be expended in development of another diagnostic if it offers promise of simplicity and usage in more of the accelerator chain.

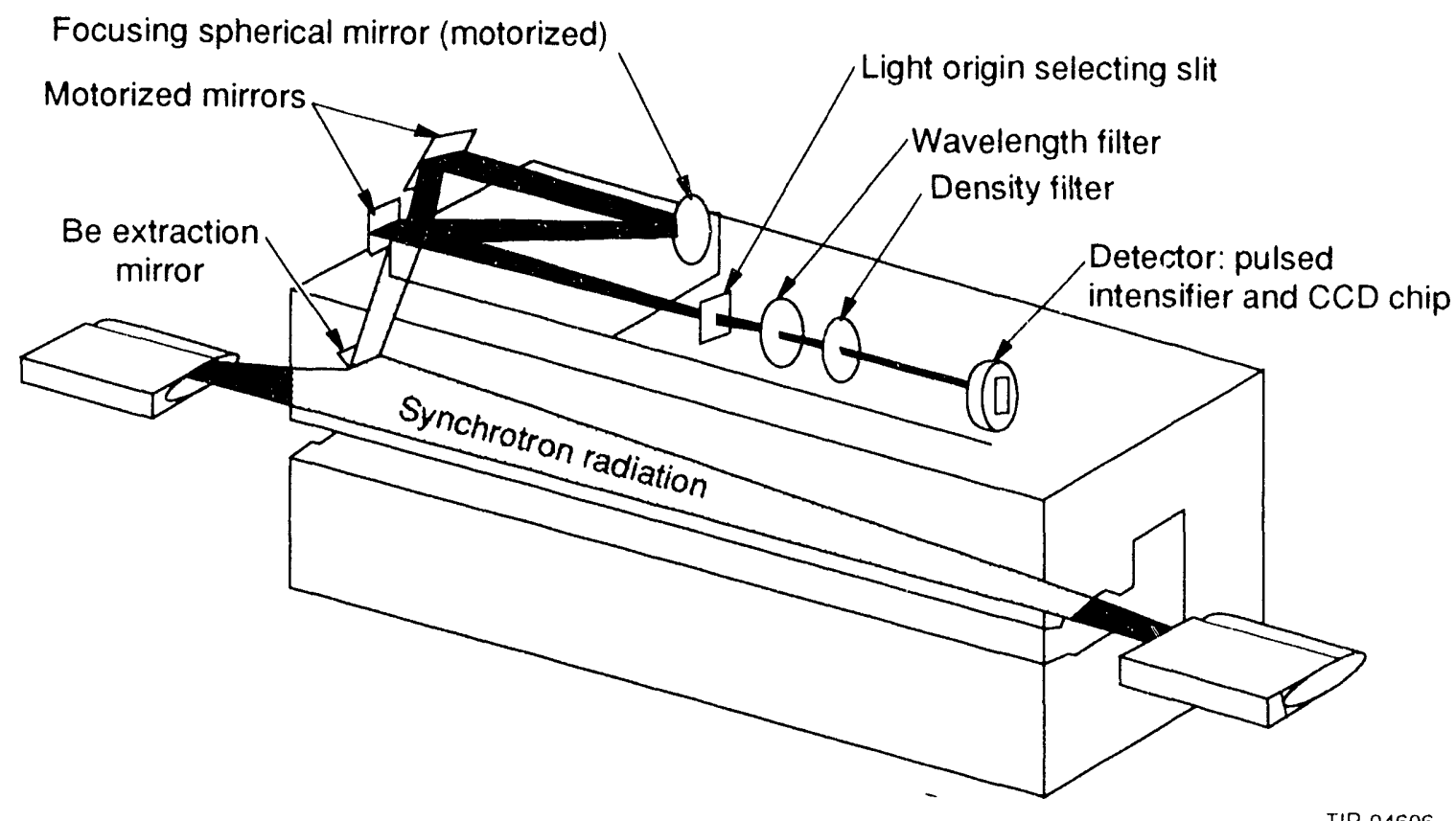

Figure 4. Simplified Schematic of SR Telescope (LEP).

\subsection{Alternative Approaches}

Table 6 lists the known and proposed alternative beam profile measurement techniques that can give minimal interference to the beam. 
Note that not all of these methods yield profiles; some are integral measurements yielding moments of the profile or are nonlinear functions of beam size and charge. Since the rms radius is the parameter commonly used for tuning accelerators and calculating luminosity, we believe that these options should not be eliminated from consideration solely because they do not furnish a profile. All of these options offer the possibility of almost continuous monitoring of the beam size. Our survey concludes that only the first four types in Table 6 hold much promise for use in the Collid r. We describe our preferred choice, the electron beam probe, below while the remainder are relegated to thin appendix.

TABLE 6. MINIMAL INTERFERENCE BEAM SIZE DIAGNOSTICS.

\begin{tabular}{|l|l|}
\hline \multicolumn{1}{|c|}{ Type } & \multicolumn{1}{|c|}{ Princlple } \\
\hline Electron beam probe & Measure deflection of probe beam by primary beam fields \\
\hline Ionization products & 1D or 2D imaging of beam produced ionization of residual gas or vapor \\
\hline Excitation light & Image light produced by beam excitation of residual gas \\
\hline Neutral beam probe & $\begin{array}{l}\text { Measure ionization of narrowly collimated neutral beam probe by primary } \\
\text { beam }\end{array}$ \\
\hline Stripline monitors & Beam quadrupole moment from quadrupole mode of wall current \\
\hline Schottky noise & Measurement of incoherent betatron noise power \\
\hline Compton scattering & Measure scattering of laser probe photons by primary beam \\
\hline Timə of flight & $\begin{array}{l}\text { Measures momentum of ionization products; maximum momentum } \\
\text { proportional to maximum field, inversely proportional to beam size }\end{array}$ \\
\hline
\end{tabular}

\subsection{Electron Beam Probe}

This diagnostic technique in its original form uses as a probe a low-energy (1-50 keV) electron beam whose diameter is small compared with the rms radius of the primary beam to be diagnosed. The basic idea is shown in Figure 5. The probe electrons are deflected as they pass near or through the primary beam, after which they are collected by a suitable detector. The deflection of the probe electrons depends on the impact parameter of the two beams, i.e., the minimum separation of the centroids of the two undeflected beams as well as on the probe beam energy and the charge distribution of the primary beam. Consequently, by measuring the deflection as a function of impact parameter, one can determine the primary beam parameters. The electron beam probe method offers the promise of being usable for measuring both radial dimensions and length of individual bunches and fine structure within the bunch. This approach to measuring beam size and charge has been used in several experiments, ${ }^{21-23}$ but has yet to be developed into a practical diagnostic. One experiment ${ }^{23}$ demonstrated the ability to respond to the fields of microbunches in an if electron accelerator, giving us confidence in being able to measure the much longer Collider bunches. In the other experiments an electron probe was used to generate profiles of charge distributions in a partially neutralized low-energy beam.

The length of a Collider proton bunch is much greater than its radius, and the particles are ultrarelativistic; consequently, any axial components of the fields are negligible when compared with the transverse components in calculating the trajectory of the probe beam. One can calculate the deflection angle in the limit that it is small enough that we can use the eikonal approximation. Tests with the computer simulation code ZBEAM, ${ }^{24}$ described in the Appendix, indicate that this is a good approximation, but for maximum accuracy the code should probably be used. In the case where the probe beam passes close to but external to the bunch charge and the impact parameter is much smaller than the beam tube radius, the deflection angle is independent both of the magnitude of the impact parameter and of the transverse charge radial distribution. ${ }^{25}$ This angle, assumed to be small, is given by

$$
\theta(b)=-\operatorname{sgn}(b) \theta(\infty)
$$




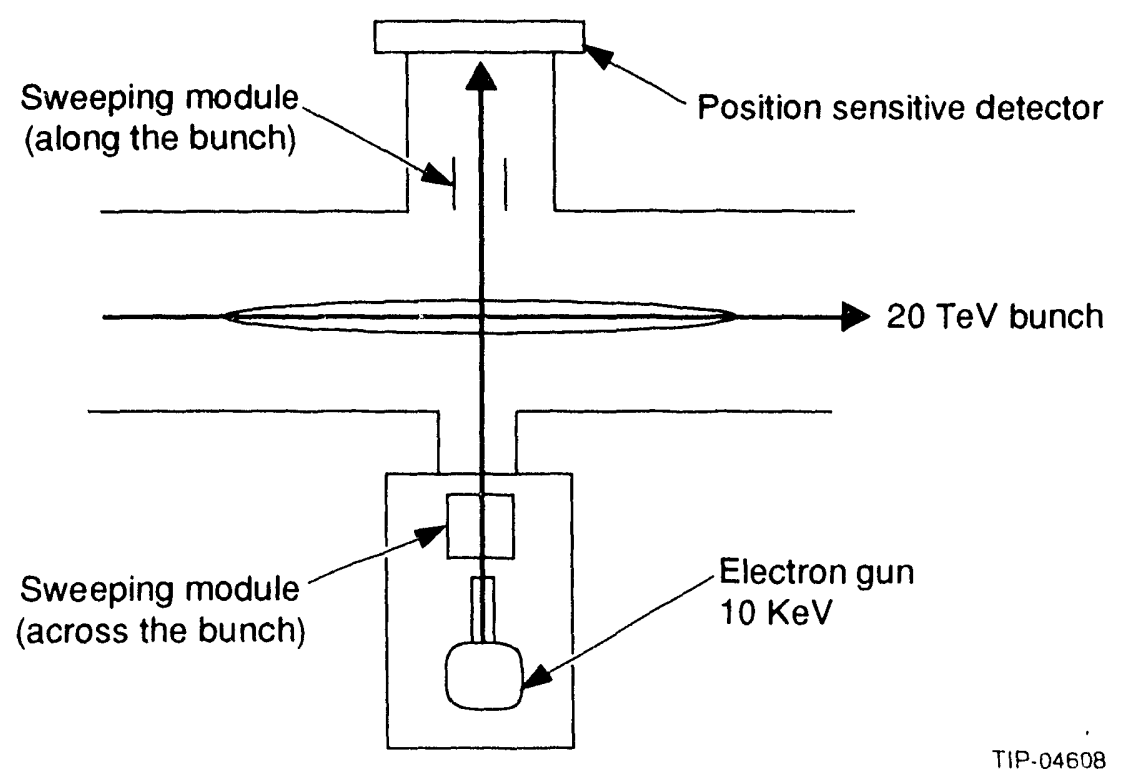

Figure 5. Schematic Diagram of the Electron Beam Diagnostic.

where $b$ is the impact parameter, $\operatorname{sgn}(b)$ is the sign function, and

$$
\theta(\infty)=\frac{\pi e^{2}\left(W+E_{0}\right)}{W\left(W / 2+E_{0}\right)} q_{L}
$$

$W$ is the energy of the probe beam, $E_{0}$ is the electron rest energy, $e$ is the charge of the probe electron (esu), and $q_{\mathrm{L}}$ is the number of protons per unit length in the $x, y$ plane of the probe beam. For reference, $\theta(\infty)=22.8 \mathrm{mrad}$ for $W=10 \mathrm{keV}$ and $q_{\mathrm{L}}=5 \times 10^{8} / \mathrm{cm}$.

When the impact parameter is smaller than the beam radius, the deflection angle is given by

$$
\theta(b)=-\theta(\infty) \int_{-\infty}^{\infty} \mathrm{d} y \int_{-\infty}^{\infty} \mathrm{d} x \varrho_{\mathrm{L}}(x, y) \operatorname{sgn}(b-x) \quad / q_{\mathrm{L}},
$$

where $\mathrm{Q}_{\mathrm{L}}(x, y)$ is the proton particle density in the $x, y$ plane containing the probe beam and

$$
q_{\mathrm{L}}=\int_{-\infty}^{\infty} \mathrm{d} y \int_{-\infty}^{\infty} \mathrm{d} x \mathrm{Q}_{\mathrm{L}}(x, y)
$$

The only $b$-dependent factor on the right hand side of Eq. (11) is $\operatorname{sgn}(b-x)$, which appears within the $x$-integrand. Since differentiating the sgn function yields a delta function,

$$
\frac{\mathrm{d}}{\mathrm{d} b} \operatorname{sgn}(b-x)=2 \delta(b-x),
$$


differentiating Eq. (11) with respect to $b$ gives

$$
\frac{\mathrm{d} \theta}{\mathrm{d} b}=\frac{-2 \theta(\infty)}{q_{\mathrm{L}}} \int_{-\infty}^{\infty} \mathrm{d} y \varrho_{\mathrm{L}}(b, y),
$$

i.e., the derivative of the deflection angle as a function of impact parameter is proportional to the $y$-integrated profile of the transverse beam particle distribution and, therefore, is similar to the signal obtained from the flying wire scanner as $b$ is varied. The electron beam probe can be used as a non-interfering flying wire scanner and profiles obtained by differentiating the data of deflection angle versus impact paramete- ${ }^{21,22}$ When used in this mode the probe beam has a number of potential advantages when compared with the flying wire scanner:

- Probe beam is non-perturbing, can be scanned slowly. It allows averaging of $\theta$ vs $b$ over all bunches, a selected batch, or a particular bunch.

- Probe beam has no moving parts subject to mechanical failure.

- Probe beam resolution is determined by the detector system; thus it is potentially better than the flying wire.

Figure 6 illustrates the differences expected in the deflection data for two density profiles with the same rms radius. If we assume a Gaussian radial bunch profile, Eq. (11) reduces to

$$
\theta(b) / \theta(\infty)=-\operatorname{Erf}\left(b / \sqrt{2} \sigma_{x}\right)
$$

where

$$
\operatorname{Erf}(z) \equiv(2 / \sqrt{\pi}) \int_{0}^{z} \mathrm{~d} t \exp \left(-t^{2}\right)=-\operatorname{Erf}(-z)
$$

and $\operatorname{Erf}(\infty)=1$.

Figure 6 shows a plot of Eq. (15); the slope at the origin is -0.7978 and the intercept of this slope with $\theta(b) / \theta(\infty)=-1$ is at $b / \sigma_{x}=1.2534$. Also plotted on the figure is the solution of Eq. (11) for an axially symmetric uniform distribution of charge. For this case the slope at the origin is -0.6355 and the intercept is at $b / \sigma_{x}=1.5736$. Experience would lead us to expect that the radial profile will be Gaussian to a good approximation. ${ }^{26}$ If we can assume that the profile is Gaussian, then the data reduction becomes simpler since one needs only the slope at the origin and $\theta(\infty)$. Individual bunches and even bunch fine structure could be analyzed by using an array of three electron beams - one of which passes external to the bunch charge while the other two penetrate the bunch with different impact parameters-to determine the first three moments of a Gaussian distribution in one of the transverse planes. A minimum of five beams would be required to measure both planes.

Such an array of probe beams could be complex and expensive; of course, only two beams would be required if one is willing to average over many bunches. However, if one desires the individual bunch measurement capability, there appears to be another possible approach. The "shadow" pattern produced at the electron detector plane when a Collider bunch interacts with a low-divergence electron probe beam whose transverse dimensions are large compared with the bunch diameter can be analyzed to yield the first three moments of a Gaussian bunch. If a probe electron is launched in a direction parallel to the $y$ axis with an initial coordinate, $x$, then using the eikonal approximation, it will strike the detector plane, located at a distance $y$ from the origin, at a point given by

$$
X=-y \theta(\infty) \text { Erf }\left((x-\varepsilon) / \sqrt{2} \sigma_{x}\right)+x,
$$




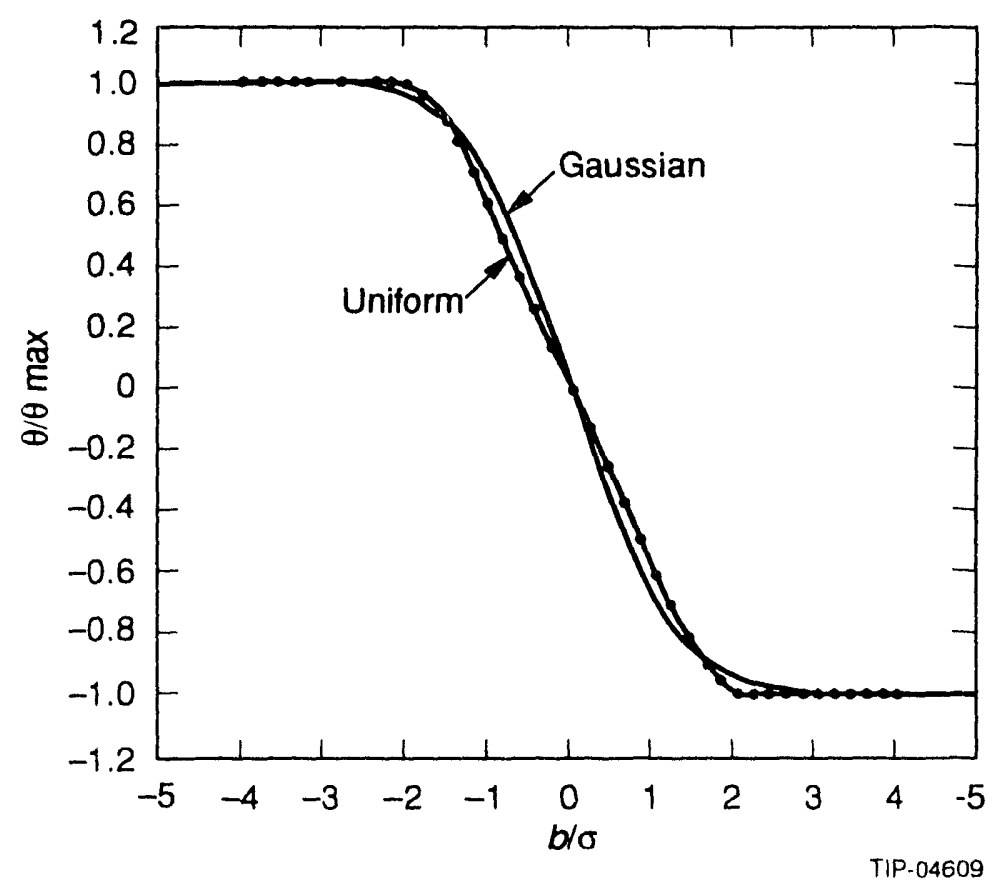

Figure 6. Deflection Angle v8. Impact Parameter, Comparing Gausslan Distribution with Uniform Distribuilon.

where $\varepsilon$ is the impact parameter between the probe beam and bunch centroids. Equation (17) can be rewritten in the form

$$
\xi \equiv X / y \theta(\infty)=-\operatorname{Erf}(t / \sqrt{2})+\zeta t+\varepsilon / y \theta(\infty)
$$

where $\zeta=\sigma_{x} / y \theta(\infty)$ and $t=(x-\varepsilon) / \sigma_{x}=b / \sigma_{x}$. In Figure 7 we have plotted $\xi$ versus $t$, with $\zeta$ as a parameter with $\varepsilon$ set to zero. As we will see below, this assumption is not necessary but it simplifies our demonstration. (The assumptions of Gaussian profile and zero angular probe beam spread do not appear to be absolutely necessary to unfold the patterns, but different assumptions would probably require the use of ZBEAM for interpretation.) The major characteristics of the shadow pattern can be determined by examining a specific case.

In Figure 8 we map a uniform density probe beam whose diameter is eight times the rms radius of the bunch to the detector plane for a case where $\zeta=0.1$. At the bottom of the figure the probe current line-density distribution is shown. The two edges of the beam, $-R$ and $R$, map to the points $X 2$ and $X 3$ in the detector plane, while the electrons with impact parameters $b 2$ and $b 5$ map to the extrema, $X 1$ and $X 4$. The impact parameter is double valued between $X 1$ and $X 2$ and between $X 3$ and $X 4$; the current between $-R$ and $b 3$ piles up between $X 1$ and $X 3$; the current between $b 4$ and $R$ piles up between $X 3$ and $X 4$, while the current between $b 3$ and $b 4$ is distributed over the wide range from $X 2$ to $X 3$. Figure 9 shows the expected relative signal density distribution at the detector plane for this case. From the rapid variation of signal density the location of $X 1, X 2, X 3$, and $X 4$ should be easily identifiable, and from these values and the knowledge of the value of the proton bunch to detector distance, $y$, the electron energy, $W$, and the undeflected probe beam radius, $R$, one can obtain $q_{\mathrm{L}}, \sigma$, and $\varepsilon$, the impact parameter between the centroids of the two beam 3 in the following manner. 


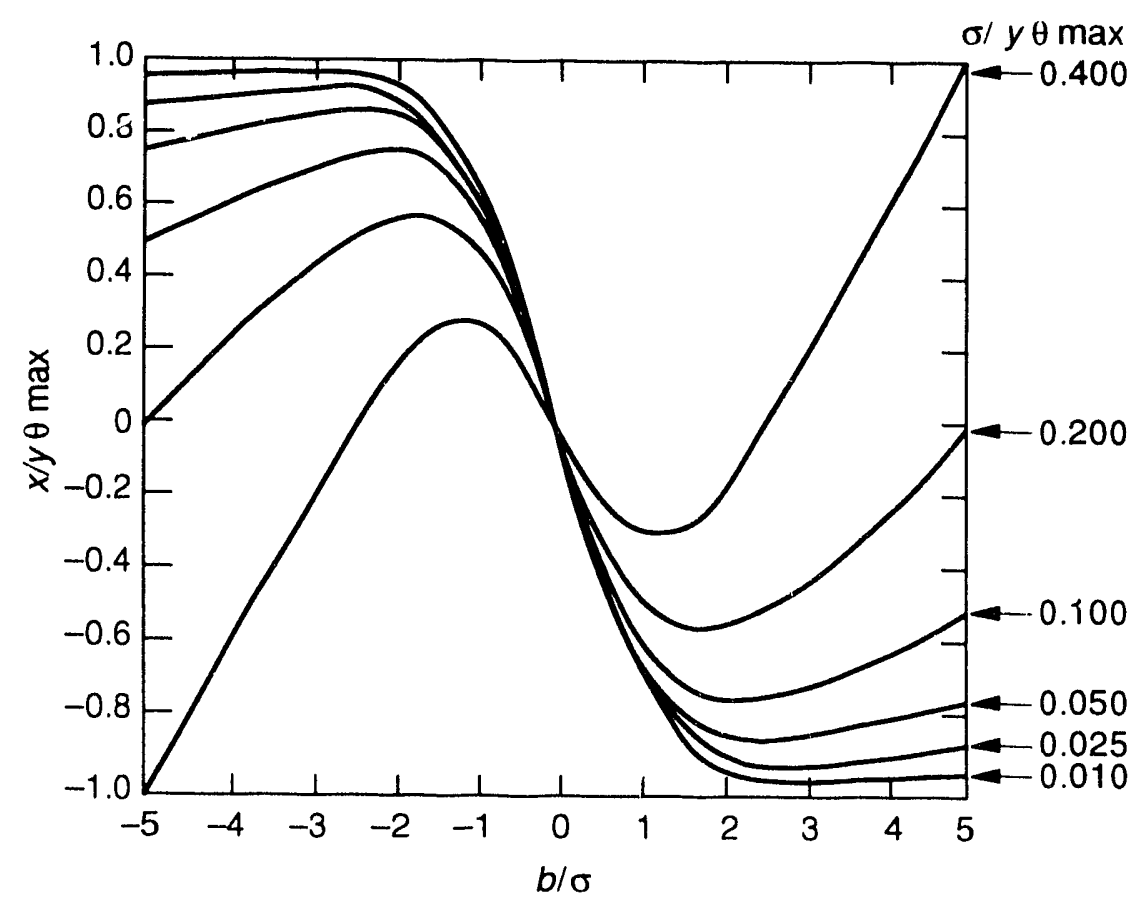

Flgure 7. Probe Electron Detector Coordinate vs. Impact Parameter.

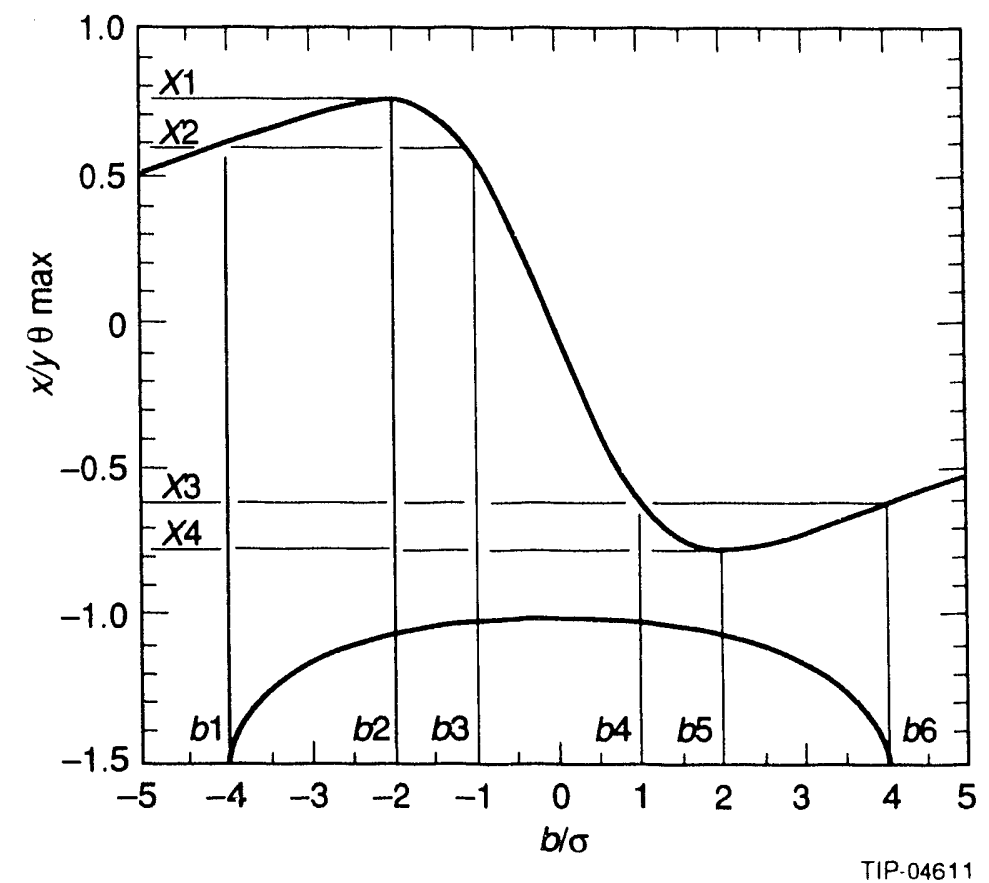

Figure 8. Map from Source Plane to Detector Plane for Wide Beam with o/y $\theta_{\max }=0.1$. 


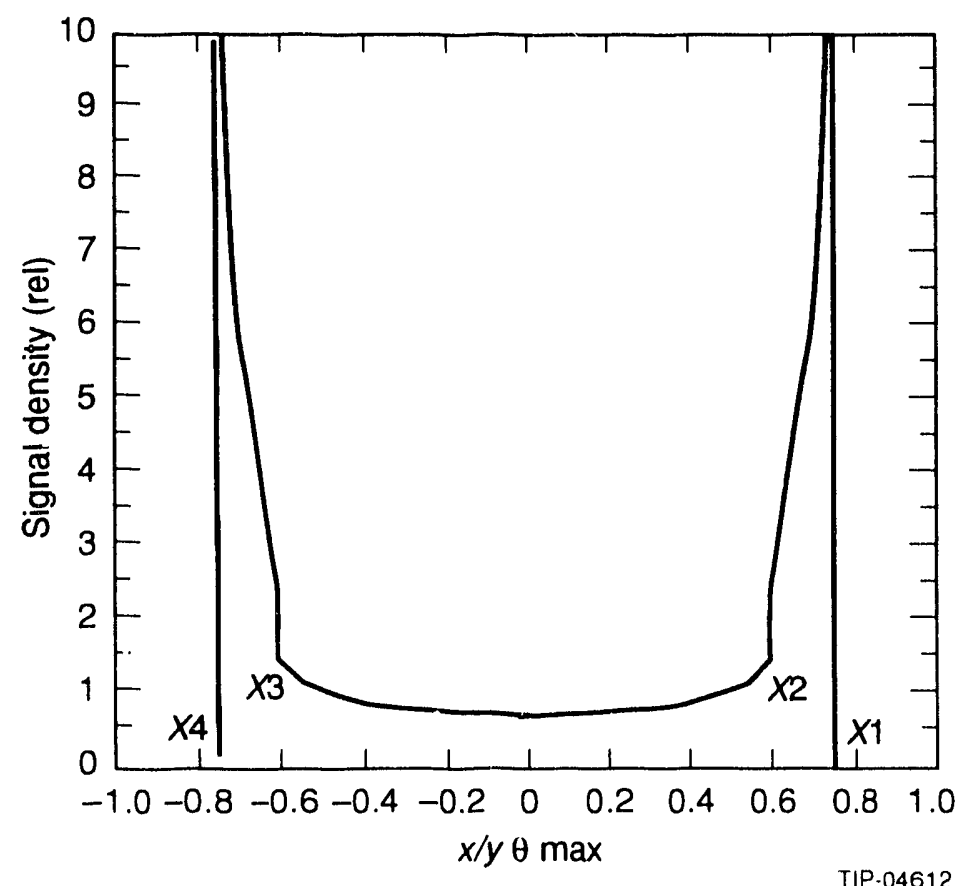

Figure 9. Relative Detector Signal Density for Wide Beam with o/y $\theta_{\max }=0.1$.

The edges of the probe beam map to the points $X 2$ and $X 3$. For $(R-\varepsilon) \gg \sigma_{x}$,

$$
\begin{aligned}
& X 2=y \theta(\infty)-R \\
& X 3=-y \theta(\infty)+R
\end{aligned}
$$

and

$$
y \theta(\infty)=(X 2-X 3) / 2+R
$$

Since $y$ and $W$ are assumed to be known, one can combine Eqs. (19) and (10) to obtain $q_{\mathrm{L}}$.

At $X 1$ and $X 4, \mathrm{~d} \xi / \mathrm{d} t=0$. Differentiating Eq. (18) and equating the result to zero, we obtain

$$
\zeta=(2 / \pi)^{1 / 2} \exp \left(-t_{m}^{2} / 2\right)
$$

and if $\xi 1=X 1 / y \theta(\infty), \xi 4=X 4 / y \theta(\infty)$, then

$$
\begin{aligned}
& \xi 1=+\operatorname{Erf}\left(t_{\mathrm{m}} / \sqrt{2}\right)-\xi_{\mathrm{m}}+\varepsilon / y \theta(\infty) \\
& \xi 4=-\operatorname{Erf}\left(t_{\mathrm{m}} / \sqrt{2}\right)+\zeta t_{\mathrm{m}}+\varepsilon / y \theta(\infty),
\end{aligned}
$$

from which

$$
\varepsilon=y \theta(\infty)(\xi 1+\xi 4) / 2=(X 1+X 4) / 2,
$$

and using Eq. (20),

$$
(2 / \pi)^{1 / 2} t_{\mathrm{m}} \exp \left(-t_{\mathrm{m}}^{2} / 2\right)-\operatorname{Erf}\left(t_{\mathrm{m}} / \sqrt{2}\right)+(\xi 1-\xi 4) / 2=0 .
$$

Equation (22) can be solved for $t_{\mathrm{m}}$, and $\sigma_{x}$ found from Eqs. (20) and (19) and the definition of $\zeta$. 
Time resolution can be obtained by sweeping the probe beam normal to its deflection plane across an imaging array. Deflection plates would be located on the opposite side of the beam tube from the gun so that the probe beam orbit through the beam tube is fixed in the absence of interactions. The suggested deflection geometry would borrow from that designed for the SSCL Linac bunch-shape monitor. ${ }^{27}$ This deflector uses a quarter-wavelength parallel-wire transmission line driven by power from the accelerator $\mathrm{rf}$ system. In effect we are creating an oscilloscope whose bandwidth is limited mainly by the interaction time, $\Delta t \approx 6 \mathrm{~b} / \mathrm{v}$, of a probe electron with the bunch field. As a bunch length monitor, a single small-diameter beam is required whose impact parameter is greater than the bunch radius. Phase resolution of less than one degree at $360 \mathrm{MHz}$ appears possible with this approach. Time resolution of the shadow patterns can also be obtained in the same manner, although perhaps not as well as with the small-diameter beam. The large-diameter beam can be focused normal to the deflection plane by biasing the deflection plates, ${ }^{27}$ thus improving the time resolution. In Figure 10 we plot the time-resolved shadow pattern expected from the passage of a baseline Collider bunch $\left(\mathrm{N}=0.75 \times 10^{10}, \sigma_{x}=173 \mu \mathrm{m}, \sigma_{t}=0.2 \mathrm{~ns}\right)$ through a 2-mm-diameter probe beam. Ultimately, interpretation of the patterns will depend on particle statistics; fortunately, we need to measure the position of rather sharp signal edges rather than the amplitude of the signals. For time-resolved single bunch shadow measurements, a well-collimated beam of the order of 1-2 mm in diameter with a current of the order of 0.1-1 mA should be satisfactory. Sources in this range are commercially available.

The resolution of the measurements of $q_{\mathrm{L}}, \sigma$, and $\varepsilon$ using the broad beam probe depends on the limits in determining $X 2$ and $X 3$, with and without the proton bunch, and $X 1$ and $X 4$. Better resolution can most likely be obtained by fitting the data in the vicinity of $X 1, X 2, X 3$, and $X 4$ to obtain more accurate values for them. The resolution will depend on specifics of apparatus design, probe beam characteristics, and choice of detector array. A modeling and experimental development program will be needed to optimize the design.

We prefer the electron beam probe technique because of its promise for furnishing a non-interfering measurement of both transverse and longitudinal profiles of individual bunches. The technique has extreme flexibility, allowing measurements over a wide range of beam parameters. The detector can be located at some radial distance from the beam tube axis, where the background radiation is somewhat reduced and where it can be shielded. It should take up no more axial space than a flying wire measurement, and it should be usable on most if not all of the chain of accelerators that make up the SSC. We strongly recommend the initiation of a development program aimed at producing an electron beam probe diagnostic usable on the SSC accelerators.

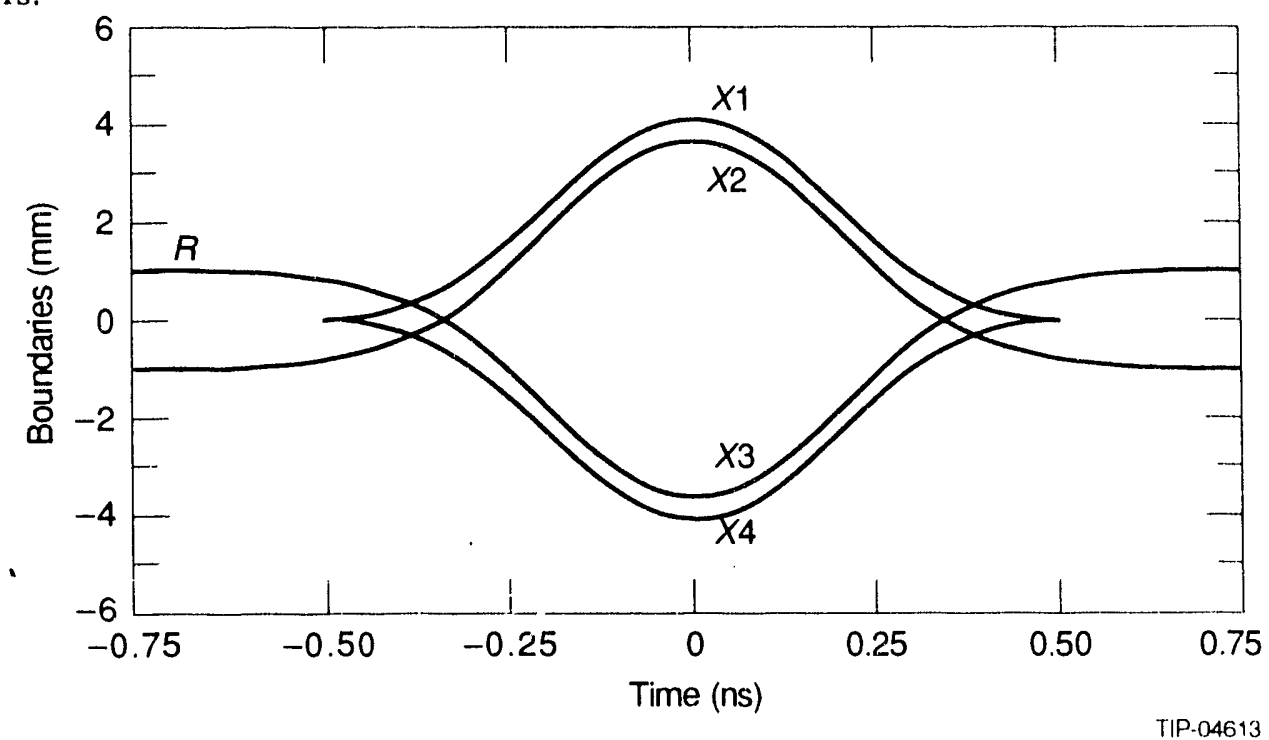

Flgure 10. 2-mm-dlameter, 10-KeV Probe Beam Interacting with Baseline Collider Bunch. Bunch-todetector distance is $200 \mathrm{~mm}$. 


\section{REFERENCES}

1. J. B. Rosenzweig et al., "Progress Towards a Turn-by-turn Beam Profile Monitor for the Fermilab Booster," AIP Conference Proceedings No. 229, Second Annual Workshop on Accelerator Instrumentation, Batavia, IL (1990), p. 328.

2. Superconducting Super Collider Laboratory, Site-Specific Conceptual Design, SSCL-SR-1056, (July 1990), p. 548.

3. J. Bosser et al., "The Micron Wire Scanner at the SPS," CERN SPS/86-26(MS), Dec. 1986.

4. J. Gannon et al., "Flying Wires at Fermilab," Proc. 1989 Part. Acc. Conf. (Chicago), pp. 68-70.

5. A. Burns et al., "Wire Scanner News from the CERN-SPS," Proc. 1989 Part. Acc. Conf. (Chicago), pp. 1580-82.

6. J. Zagel et al., "Upgrades to the Fermilab Flying Wire Systems," Proc. 1991 Part. Acc. Conf. (San Francisco), pp. 1174-76.

7. X. Q. Wang et al., "Design and Commissioning of Flying Wires in the Fermilab Accumulator," Proc. 1991 Part. Acc. Conf. (San Francisco), pp. 1180-82.

8. B. Bouchet et al., "Wire Scanners at LEP," Proc. 1991 Part. Acc. Conf. (San Francisco), pp. 1186-88.

9. M. C. Ross et al., "Wire Scanners for Beam Size and Emittance Measurements at the SLC," Proc. 1991 Part. Acc. Conf. (San Francisco), pp. 1201-03.

10. LHC Study Group, "Design Study of the Large Hadron Collider (LHC)," CERN 91-03 (2 May 1991) pp. 137-38.

11. R. Bossart et al., "Observation of Visible Synchrotron Radiation Emitted by a High-Energy Proton Beam at the Edge of a Magnetic Field," Nucl. Instr. Meth. 164 375-380 (1979).

12. R. Bossart et al., "Proton Beam Profile Measurements with Synchrotron Light," CERN/SPS/80-8 (ABM).

13. A. A. Hahn and P. Hurh, "Results from a Prototype Beam Monitor in the Tevatron using Synchrotron Light," Proc. 1991 Part. Acc. Conf. (San Francisco), pp. 1177-79.

14. A. A. Hahn and P. Hurh, "Results from an Imaging Beam Monitor in the Tevatron using Synchrotron Light," Proc. XVth Int. Conf. H. E. Accel. (HEACC'92) Hamburg (July 20-24, 1992).

15. A. P. Sabersky, "The Geometry and Optics of Synchrotron Radiation," Part. Accel. 5 199-206 (1973).

16. R. Coisson, "Angular-spectral Distribution and Polarization of Synchrotron Radiation from a 'Short' Magnet," Phys. Rev A 20 524-28 (1979).

17. J. Bosser et al., "Possible Uses of Synchrotron Radiation for Electron Beam Profile Monitoring in LEP and SPS," CERN/SPS/ABM LEP Note 397 (1982).

18. A. Hofmann and E. Meot, "Optical Resolution of Beam Cross-Section Measurements by Means of Synchrotron Radiation," Nucl. Instr. Meth. 203 483-493 (1982).

19. A. Hofmann, "Theory of Synchrotron Radiation," SSRL ACD-Note 38 (1986). 
20. J. Bosser et al., "Preliminary Studies on a Profile Monitor for the LHC Using Synchrotron Radiation," CERN LHC/ Note 192 (6/17/92).

21. V. Shestak et al., "Electron Beam Probe for Ion Beam Diagnostics," TRIUMF Design Note, TRI-DN-87-36 Rev. a (1987).

22. P. Gross et al., "An Electron Beam Probe for Ion Beam Diagnosis," Proc. 1990 Europ. Part. Acc. Conf., (EPAC '90), pp 806-08.

23. J. A. Pasour and M. T. Ngo, "Nonperturbing Electron Beam Probe to Diagnose Charged-Particle Beams," Rev. Sci. Instrum. 63 3027-39 (1992).

24. E. Tsyganov and A. Zinchenko, "ZBEAM, charge tracing code for the SSC environment," in publication.

25. E. Tsyganov et al., "Electron Beam Emittance Monitor for the SSC," SSCL-Preprint-179, (December 1992).

26. D. A. Edwards and M. J. Syphers, An Introduction to the Physics of High Energy Accelerators, John Wiley \& Sons, Inc. (1992), p. 263.

27. A. V. Feschenko and S. K. Esin, "Bunch Shape Monitor for the SSCL Linac, Preliminary Design Review Report," Acad. Sci. Russia, Inst. of Nucl. Research, (Sept. 1992). 


\section{APPENDIX \\ ALTERNATE TECHNIQUES CONSIDERED}

\section{Ionization Products Imaging}

The imaging of ionization products produced by interaction of high-energy beam particles with residual gas, ${ }^{\mathrm{A} 1-\mathrm{A} 3}$ or with curtains of injected material, ${ }^{\mathrm{A} 4}, \mathrm{~A} 5$ has been used for monitoring the beam profile at many accelerators. In fact, this approach is being taken to obtain beam profiles on a turn-by-turn basis on the Fermilab Booster. ${ }^{1}$ A strong electric field oriented normal to the beam path transports the secondary ions to an imaging array, where a projection of the radial intensity of ion production is detecred. Figure A.1 illustrates the geometry.

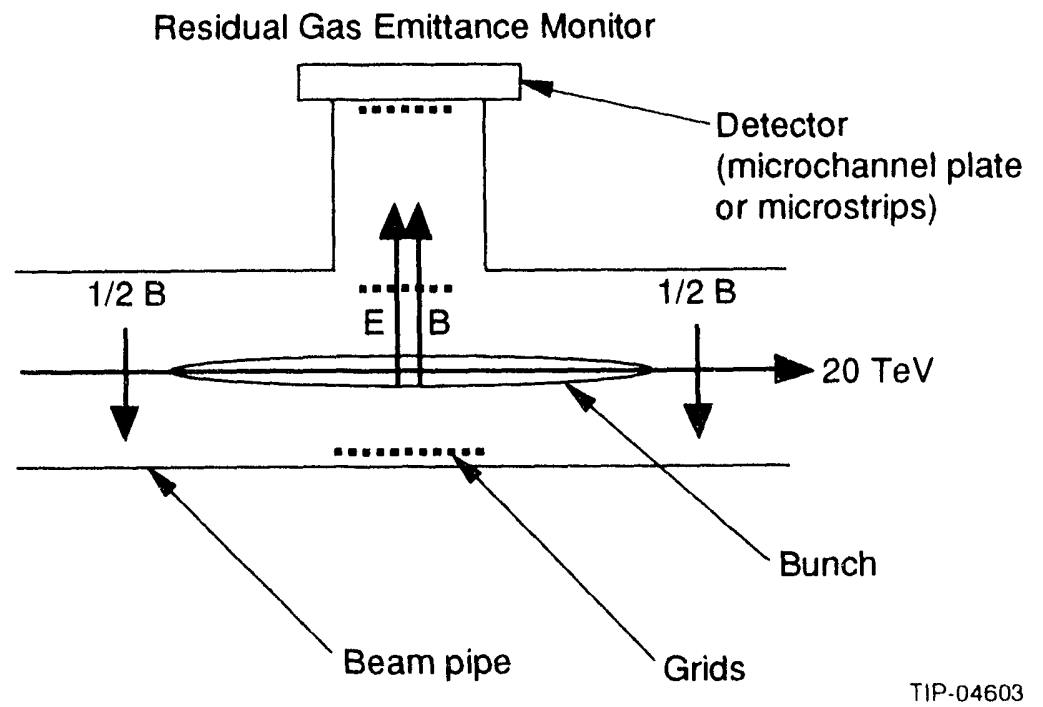

Figure A.1. Ionization Products Imaging Geometry.

For an unbaked vacuum system the residual gas will be a mixture mainly of $\mathrm{CO}, \mathrm{H}_{2} \mathrm{O}$, and $\mathrm{N}_{2}$. Any of these has a cross section for ionization by ultrarelativistic ions of $\sim 2 \times 10^{-18} \mathrm{~cm}^{2}{ }^{A 6}$ The number of ion pairs produced per centimeter length of beam path per second by the SSC Collider beam is

$$
\mathrm{d} N / \mathrm{d} t=(I / q) n_{\mathrm{g}} \sigma_{\mathrm{i}},
$$

where $I$ is the beam current, $q$ is the proton charge in coulombs, $n_{\mathrm{g}}$ is the residual gas density in molecules per cc, and $\sigma_{i}$ the ionization cross section. For a warm tube pressure of $10^{-9}$ torr and a beam current of $0.072 \mathrm{~A}$, we estimate that $\mathrm{d} N / \mathrm{d} t$ is of the order of $3 \times 10^{7} \mathrm{ion}$ pairs $/ \mathrm{cm} / \mathrm{s}$. In the absence of image spreading, the peak particle current density on the imaging array will be $\sim 7 \times 10^{10} / \mathrm{cm}^{2}$ s for a Gaussian distribution with $\sigma=173 \mu \mathrm{m}$. If we assume collecting strips $50 \mu \mathrm{m}$ wide by $1 \mathrm{~cm}$ in length, the maximum number of primary ions per strip per turn will be $\sim 1 \times 10^{5}$. The expected fractional statistical fluctuation in this signal is $\sim 0.3 \%$. If better statistics or time resolution is desired, the local pressure could be raised. If the local pressure is raised to $10^{-6}$ torr, the maximum number of primary ions to the same-sized collector strip will be $\sim 6 \times 10^{3}$ per single bunch, and the expected statistical fluctuation will be $\sim 1 \%$.

Distortion of the image will be caused by the beam space charge; usually this spreading has not been important because the beams were much larger than those expected in the SSC, and often the images were used only for qualitative indicators of beam presence. We have used a computer simulation code, ZBEAM, ${ }^{24}$ 
to model the behavior of primary electrons produced by beam ionization when external electric and magnetic fields are superimposed on the space charge field of a bunch. The bunch is represented by 2907 line charges distributed with a two-dimensional Gaussian density distribution. Total charge of the bunch is taken equal to $10^{10}$ electron charges, the length of the bunch is $10 \mathrm{~cm}$, and to simulate the passage of the bunch, the electrons move under the influence of the bunch for only the first $0.3 \mathrm{~ns}$ after which they drift in the background fields to the detector plane in about $0.5 \mathrm{~ns}$. The time step is $0.001 \mathrm{~ns}$. The beam direction is along the $Z$ axis; the external fields directed along the $Y$ axis and electrons launched with various values of $X$ and $Y$ are followed until they reach the plane $Y=20 \mathrm{~mm}$.

Initial runs with beam $\sigma_{x}=\sigma_{y}=50 \mu \mathrm{m}$ and external electric field of $10 \mathrm{kV} / \mathrm{cm}$ showed spreading of the electron image much greater than the size of the beam. The use of a strong magnetic field parallel to the electric field would be expected to greatly reduce the spreading since the ions would be bound to the field lines. (Magnets for producing such a field have been incorporated in the HERA ionization profile monitors $\mathrm{A}^{7}$ but have not been used. ${ }^{A 8}$ ) Figure A.2 shows the trajectory of an electron with zero initial velocity at $X=100 \mu \mathrm{m}, Y=0$ in fields $E_{Y}=10 \mathrm{keV} / \mathrm{cm}, B_{Y}=1 \mathrm{~T}$ plus the bunch field. The electron drifts in the strong radial potential well of the beam until the bunch field is turned off, after which it spirals out to the $Y=20 \mathrm{~mm}$ plane. The maximum Larmor radius is of the order of $20 \mu \mathrm{m}$, corresponding to a maximum energy component normal to the external fields of the order of $40 \mathrm{eV}$. Taking a value of $\sigma_{x B} \approx 15 \mu \mathrm{m}$ for the rms spread along the $X$ axis, the image of a $\sigma_{x}=50 \mu \mathrm{m}$ beam would have $\sigma_{x l}=52 \mu \mathrm{m}$, an increase of $4 \%$.

Reducing the electric field to $1 \mathrm{kV} / \mathrm{cm}$ had little effect on the image spreading in a 1-T magnetic field, but lowering the magnetic field did, as should be expected. We can lower the magnetic field somewhat if we measure at the utility dogleg location where the beam rms radius is of the order of $150 \mu \mathrm{m}$. If we assume that the electron will have an energy perpendicular to the magnetic field of the order of $50 \mathrm{eV}$ from a combination of its initial kinetic energy and the contribution of the beam field, then a field of the order of $0.25 \mathrm{~T}$ will keep the spreading of the image to less than $10 \%$. By comparing the images with profiles obtained from flying wire measurements, it should be possible to unfold the $\sigma_{x B}$ due to finite electron orbits and incorporate these values into algorithms for correcting the images.

Compensating electric and magnetic fields anti-parallel to the detector fields will be required to nullify the ir perturbation of the beam. Since both a vertical and horizontal profile are required, the compensation is done most efficiently by orienting a magnetic field and an electric field of the proper strengths to cancel the resultant of the horizontal and vertical fields. If these fields are also used to form an image, beam tomography will be possible.

The addition of the magnetic field makes this diagnostic a strong competitor for the job of beam profile monitoring. The rather slow variation of ion pair production with energy means that the diagnostic has potential for use in all of the SSC accelerators and transfer lines; the task should be easier at lower energies, where the beam is larger. The chief drawback of this approach is the complexity introduced by the addition of the magnetic field. Because of the required magnetic field strength, iron-core electromagnets or permanent magnets will most likely be required. Detector arrays would have to be mounted on the magnet faces. This may limit the choice of detectors because of interference with the magnet pole pieces or the associated field. In addition, the detector will be close to the beam and most likely in a strong radiation field. Although measurements of individual bunches may be possible, time resolution within the bunch does not seem likely. A group at INP has proposed building an ionization profile monitor with a $0.2-\mathrm{T}$ field for the SSC LEB. ${ }^{\mathrm{A} 9}$ At the present time this proposal has not been accepted; instead, a residual gas ionization detector without magnetic field is planned for the LEB. ${ }^{\mathrm{A} 10}$ 

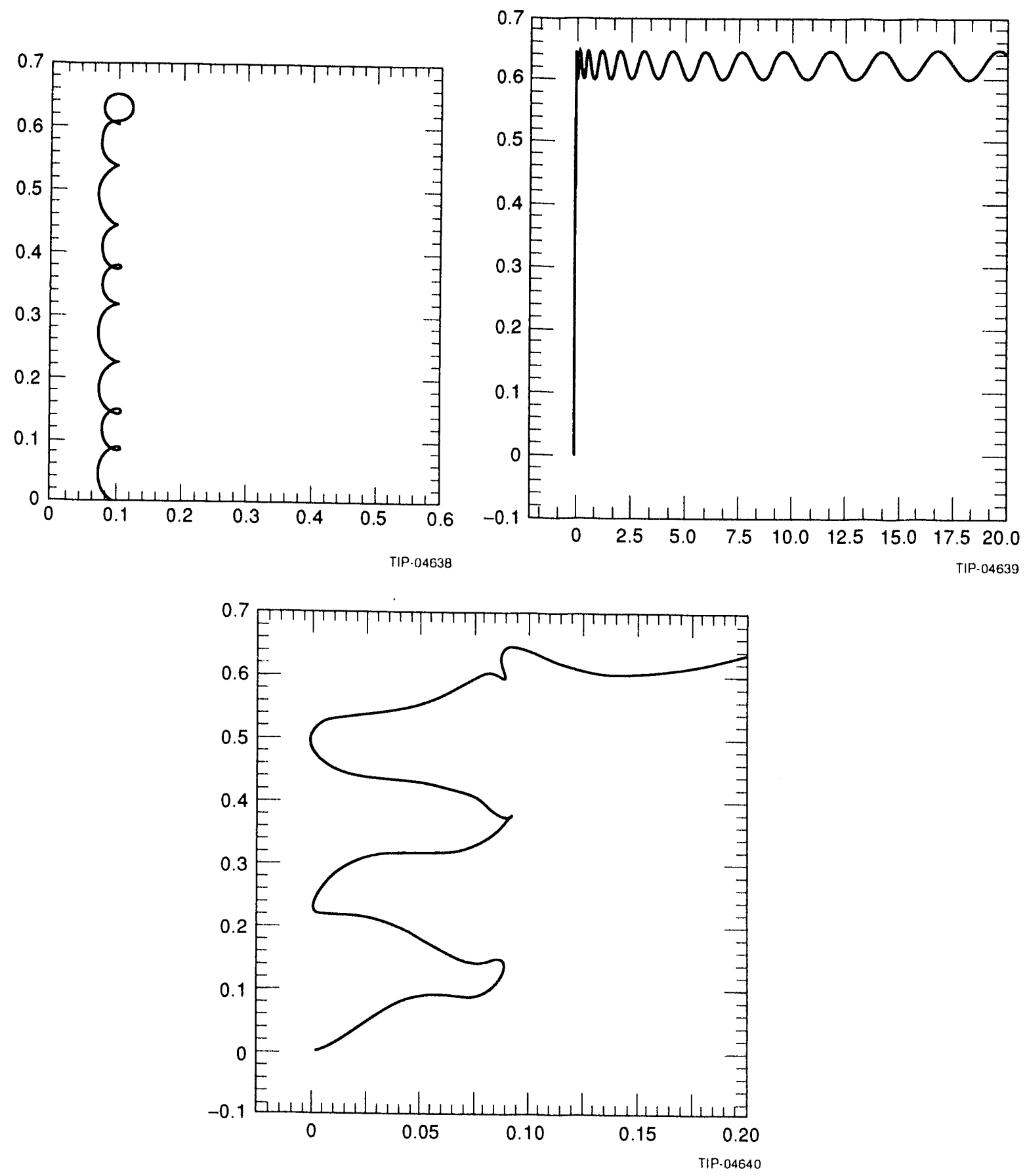

Figure A.2. Trajectory of Electron in Beam Plus External Fields.

\section{Excitation Light Imaging}

The imaging of the light produced by excitation of residual gas by the passage of a beam has been used for radial profile measurements of relatively low-energy beams. ${ }^{\mathrm{A} 11-\mathrm{A} 13}$ This technique has the attraction that it is non-perturbing, the image can be viewed from multiple directions at a single axial position, and the image is not diffraction-limited. 
We estimate the applicability of this technique to the Collider beam by making the assumption that the optical excitation probability varies with energy in a similar manner as the ion pair production. An energetic proton in air loses approximately $95 \mathrm{eV}$ per primary ion pair produced. This energy goes into the ionization energy, the kinetic energy of the product ion pair, and any photons produced in the process. Most of the energy can be accounted for in the ionization energy and the kinetic energy; consequently, one would estimate that at most only a few detectable photons are produced per event. If we assume that the order of one detectable photon is emitted for each ionization event, then we would estimate a source strength of the order of $5 \times$ $10^{7}$ photons $/ \mathrm{cm} \cdot \mathrm{s}$ for the conditions described in the previous section. The disadvantage of this approach becomes apparent when we compare the efficiency of imaging these photons with that of imaging the ionization products. In the latter case, the detection efficiency is close to one, while with the former the combination of the fraction of the photons that can be reasonably be collected by an imaging lense or mirror $(\sim 1 \%)$ with the quantum efficiency of the image intensifier photo-cathode $(\sim 10 \%)$ leads to a detection efficiency of the order of $0.1 \%$. It will take of the order of a thousand times longer to form an optical image than to form an image of the ionization products under equivalent conditions. To do even this well would probably require the use of a lense close to the source and, therefore, in the intense radiation field of the Collider beam losses. We conclude that this is not an attractive approach for the Collider because of its low sensitivity.

\section{Neutral Beam Probe}

The use of a thin $(\sim 10 \mu \mathrm{m})$ beam of $10-\mathrm{KeV}$-energy, cesium-neutral atoms as a probe has been suggested. ${ }^{A 14}$ The ionization of the probe beam by the main beam would be measured as the probe beam is swept through the main beam. The geometry is shown in Figure A.3.

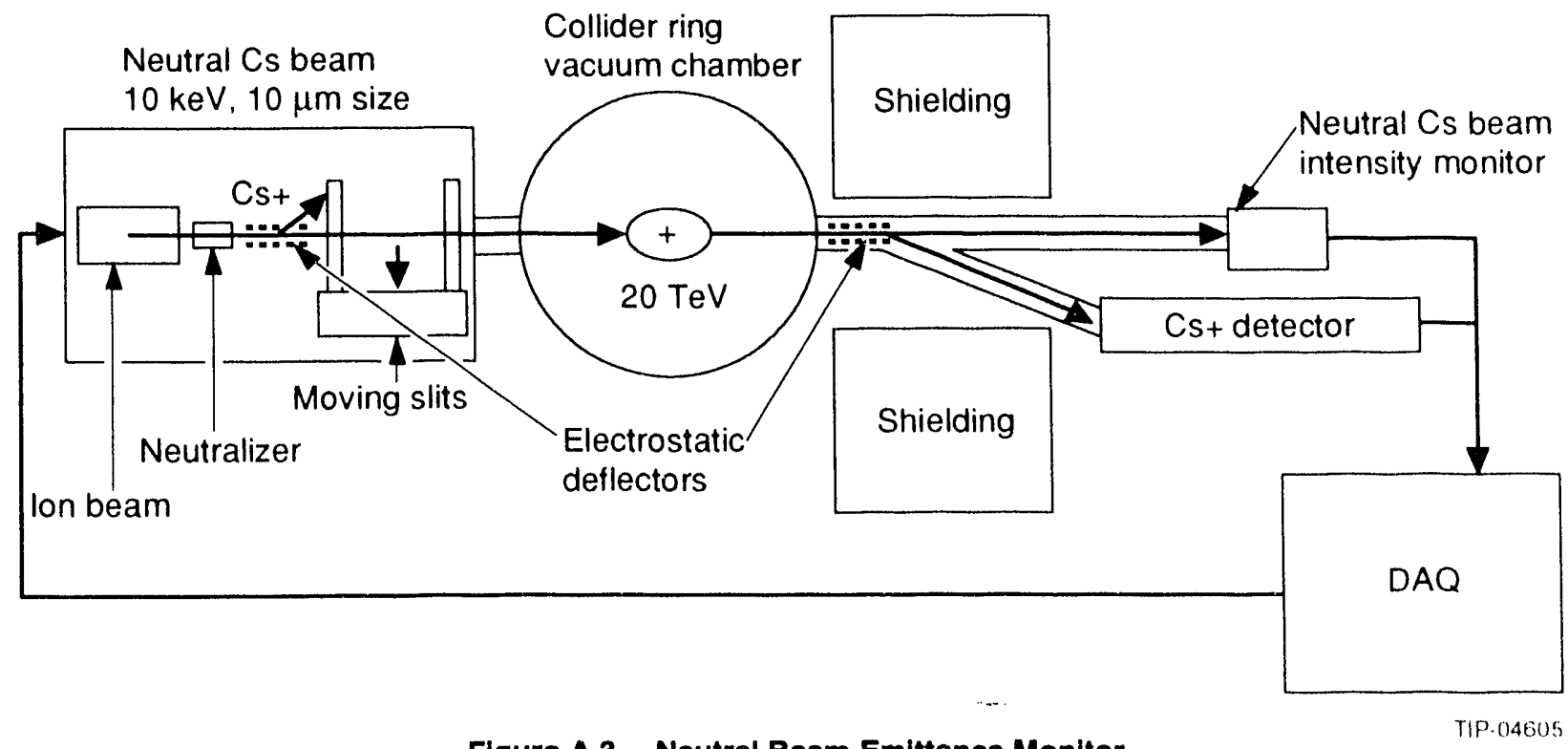

Figure A.3. Neutral Beam Emittance Monitor.

It is possible to extract cesium from a source with a current density of $5 \mathrm{~mA} / \mathrm{cm}^{2}$ so a slit $1 \mathrm{~cm}$ long and $10 \mu \mathrm{m}$ wide will pass a flux of $\sim 3 \times 10^{13} \mathrm{ions} / \mathrm{s}$. The angular divergence is limited by the effective temperature of the source; for cesium hot plate sources, this has been measured to be approximately equal to the plate temperature, or around $0.1 \mathrm{eV}$. Therefore the angular divergence of a properly designed $10-\mathrm{keV}$ beam will be around $3 \mathrm{mrad}$. If an identical slit is located $10 \mathrm{~cm}$ downstream and parallel to the first slit, the passing current will have an angular spread of about $0.1 \mathrm{mrad}$ and a flux of about $10^{12}$ atoms $/ \mathrm{s}$, assuming $\sim 90 \%$ resonant 
charge exchange neutralization in a cesium vapor neutralizer between the slits. The angular spread caused by the resonant charge exchange event is negligible compared with the 3-mrad natural divergence of the beam.

If the final slit is located $10 \mathrm{~cm}$ from the beam axis, it will have a fwhm of $\sim 20 \mu \mathrm{m}$ at the beam plane and will produce negligible spreading of a measured profile. The increase in angular spread of the ions due to the space charge of the bunch is negligible compared with the initial spread of the incident atoms; consequently, one expects that a detector could be located at some distance from the target without appreciable loss of signal. Assuming an ionization cross section of $\sim 2 \times 10^{-17} \mathrm{~cm}^{2}$, a beam sigma of $\sim 50 \mu \mathrm{m}$, and a baseline current of $72 \mathrm{~mA}$, the ionization fraction of the Cs beam will be $\sim 6 \times 10^{-5}$ and the peak count rate can be of the order of $6 \times 10^{7}$ ions/s. Many radial scans per second would be possible with this counting rate.

The proof of principle of this concept would lie in showing that a beam of the above characteristics can actually be produced. A technical disadvantage is that scanning requires mechanical motion of the slits, although only over very small distances, and that the slit must be maintained parallel to the axis of the beam to within of the order of a milliradian to avoid image broadening. On the other hand, this diagnostic may be made less sensitive to beam-induced radiation than most of the others considered because the detector may be located some distance from the beam tube axis.

Other energetic neutral beams and even supersonic microjets of gas could be used instead of the cesium beam. The latter approach offers the possibility of both greatly increasing the magnitude of the signal and simplifying the measurement when compared with the energetic neutral beams. As with the energetic neutral beam, the proof of principle for the microjet approach is to demonstrate that probe gas beams with the necessary parameters can be produced.

While this approach has certain attractions, we have eliminated it from present consideration because the necessity of mechanical scanning makes fast measurement of individual bunch profiles difficult and the ability to produce the required beams or jets has yet to be demonstrated.

\section{Measurement of Beam Quadrupole Moment}

The signals from the four striplines of a two-plane BPM can be combined to measure the quadrupole moment of the wall current in the beam pipe. This component is a function of the quadrupole moment of the beam, $\left(\sigma_{x}^{2}-\sigma_{y}^{2}\right)$ A $15, A 16$ The SLC scheme for the measurement is shown in Figure A.4. Using the notation of the figure where $N, S, T, B$ are the signals from the four striplines, the expression for the quadrupole moment is

$$
q \equiv \frac{(N+S)-(T+B)}{N+S+T+B}=2\left[\frac{\sigma_{x}^{2}-\sigma_{y}^{2}}{a^{2}}+\frac{x^{2}-y^{2}}{a^{2}}+\text { higher order terms }\right],
$$

where $a$ is the beam tube radius and $x, y$ are the beam centroid positions in the BPM coordinate system:

$$
\begin{aligned}
& x \cong a \frac{N-S}{N+S+T+B} \\
& y \cong a \frac{T-B}{N+S+T+B}
\end{aligned}
$$

The resolution of the BPM is defined as the minimum change in centroid position that can be detected:

$$
R=a \frac{\Delta}{N+S+T+B},
$$


where $\Delta$ is the smallest signal change that can be detected. Assuming that $\left(\sigma_{x}^{2}-\sigma_{y}^{2}\right)>\left(x^{2}-y^{2}\right)$, the resolution of the $\sigma_{x}$ measurement is given by

$$
\delta \sigma_{x}=\left[\frac{a R}{2\left(1-\beta_{y} / \beta_{x}\right)}\right]^{1 / 2},
$$

and solving for $R$, the BPM resolution required to measure $\sigma_{x}$ with maximum error $\delta \sigma_{x}$ is

$$
R<2 \frac{\delta \sigma_{x}^{2}}{a\left(1-\beta_{y} / \beta_{x}\right)}
$$

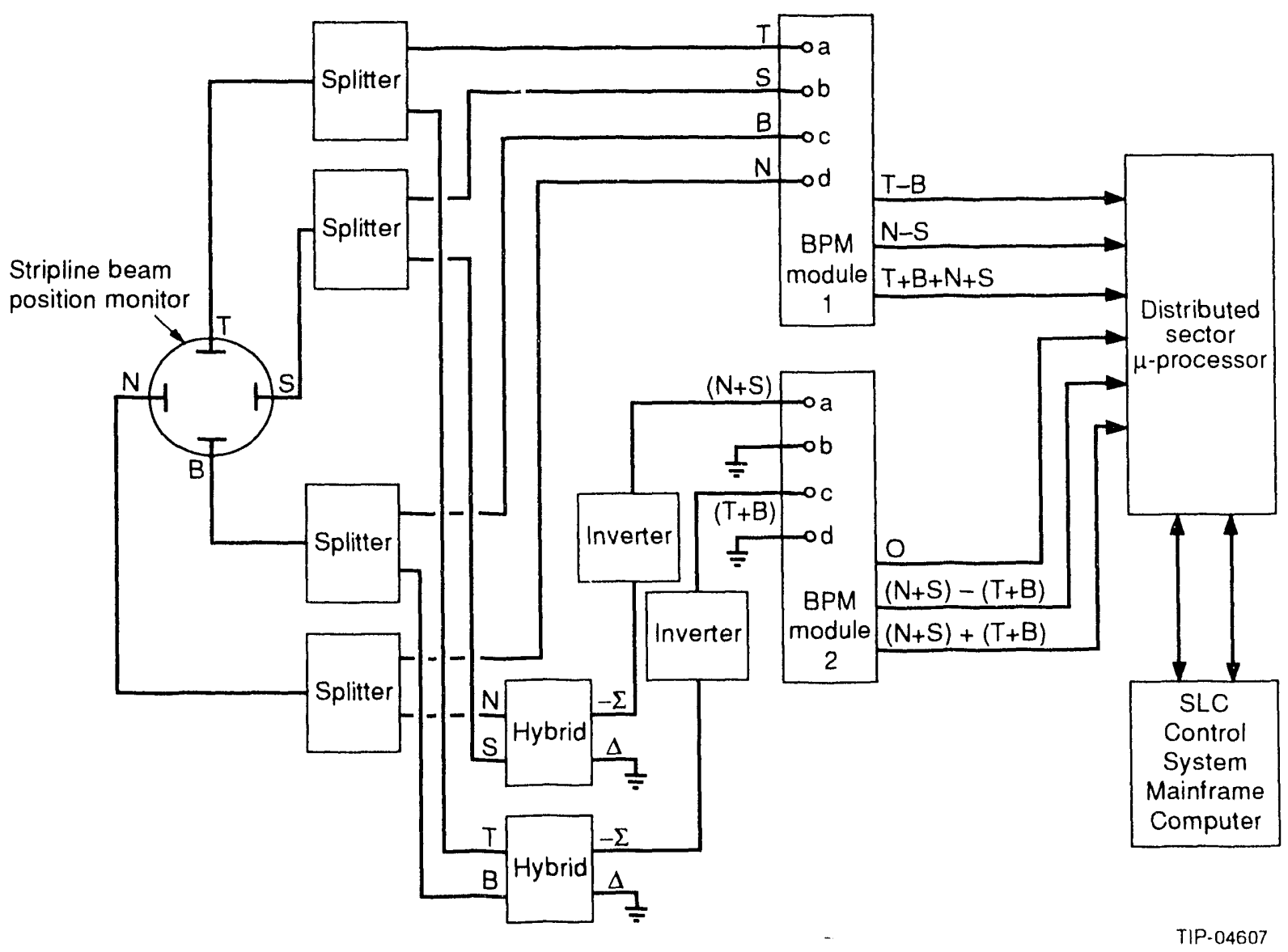

Figure A.4. Scheme for Measuring Beam Quadrupole Moment.

In the utility straight section, where $\beta_{y} / \beta_{x}=0.13$ and $\sigma_{x}=129 \mu \mathrm{m}$ at $20 \mathrm{TeV}$ for baseline conditions, we require $\delta \sigma_{x}<6 \mu \mathrm{m}$ for $\pm 5 \%$ error in the $\sigma_{x}$ measurement. This in turn requires a BPM resolution $R$ of $5 \mathrm{~nm}$, an impossibly difficult requirement.

While the approach outlined in Reference A17 is somewhat different, relying on the Fourier analysis of the azimuthal distribution of the wall currents measured by 16 striplines uniformly spaced around the periphery of the beam tube wall, there is no reason to believe it will be much more sensitive in determining $\sigma_{x}$.

\section{Schottky Transverse Noise Measurement}

The analysis of beam Schottky noise can yield important information about the beam, one item of which is, in theory, the beam rms radius. ${ }^{\mathrm{A} 18, \mathrm{~A} 19}$ To determine the size one must make an absolute measurement of the 
power in a betatron sideband of the Schottky spectra. Transverse Schottky signals represent the sum of the incoherent contributions of all the particles in the beam; for a beam of $N$ particles, the rms power is proportional to $N \sigma_{x}{ }^{2}$. If the noise measurement is made in a frequency range below the single bunch frequency $(\sim 800 \mathrm{MHz})$ and the particles have any coherent motion, the $N$-particle signal will be $N$ times that of a single particle, and the rms coherent power will be proportional to $N^{2} x^{2}$, where $x$ is the centroid displacement. In this case for the signal of the coherent displacement of the beam not to mask the incoherent Schottky noise signal, it is necessary that $x<\sigma_{x} /(N)^{1 / 2}$. For $N \approx 10^{10}$ and $\sigma_{x} \approx 100 \mu \mathrm{m}$, the coherent motion must be kept below $1 \mathrm{~nm}$ in order to observe the Schottky component. In theory this problem can be bypassed by measuring in a frequency band much higher than the single-bunch frequency. Measurement of the incoherent Schottky noise in the Collider appears very difficult, however, and we do not regard this as a promising approach to follow for beam size measurements.

\section{Compton Scattering}

A beam size measuring system for ultra-small electron beams utilizing a laser interferometer is now under construction. ${ }^{\mathrm{A} 20}$ Two crossing laser beams form interference fringes that cause spatial periodic modulation in the total flux of Compton-scattered $\gamma$-rays. The transverse spot size of the electron beam can be determined from the modulation depth. Since this is apparently a difficult experiment to do with electrons and the cross section for Compton scattering off protons is down by $\left(\mathrm{m}_{\mathrm{e}} / \mathrm{m}\right)^{2}$ from the electron value, this approach does not appear feasible.

\section{Time of Flight}

This scheme is still under development and is intended to measure the beam dimensions at the focal point of the Final Focus Test Beam (FFTB) at Stanford Linear Accelerator Center. ${ }^{A 21,}$ A22 The goal is to measure a spot size of about $1 \mu \mathrm{m}$ horizontally, which can be vertically decreased down to $60 \mathrm{~nm}$ in a flat beam mode of operation. The principle of the measurement is based on the transverse kick given to ions by the space charge of the beam. The ions are produced by ionization of a pulsed gas target at the focus. In the experiment described, argon ions receive a kick proportional to the electric space-charge field. The maximum velocity of these ions is proportional to the maximum field, which is inversely proportional to the beam dimensions. The time of flight for the ions to reach a detector has a minimum value that scales linearly with the radius of a round beam. For a flat beam, the time of flight is also slightly dependent on the beam aspect ratio. A second measurement with helium gas will allow one to obtain the aspect ratio and resolve the ambiguity of the first measurement. The light helium ions are trapped and oscillate in the space-charge field during the passage of the electron beam pulse. In the case of a flat horizontal beam, the averaged oscillation amplitude is larger in the horizontal direction than in the vertical one. After passage of the beam the ions are emitted in the transverse plane with an angular distribution peaked along the horizontal direction. The anisotropy of the angular distribution will then give the beam aspect ratio.

While this technique may be useful (it still must be tested) for measuring very small beams with low bunch-repetition rate, the time of flight technique is not useful for Collider beam sizes and bunch-repetition rates. A simple calculation supports this conclusion. Assuming a beam with a Gaussian radial profile, an ion formed at radius $r$ will have an average radial momentum kick,

$$
<p>=\left(\frac{N e^{2}}{2 \pi \varepsilon c}\right) \frac{\left(1-\exp \left(-r^{2} / \sigma^{2}\right)\right)}{r},
$$

where $N$ is the number of beam protons per bunch. This expression has a maximum for $r \approx 1.58 \sigma$, where

$$
<p\rangle_{\max }=0.69 \times 10^{-36}(\mathrm{~N} / \mathrm{\sigma}) \mathrm{kg}-\mathrm{m} / \mathrm{s} .
$$

For a $10^{10}$ particle bunch with a rms radius of $50 \mu \mathrm{m}$, the peak velocity kick given to an ion of mass number $A$ is of the order of $\left(8 \times 10^{4} / A\right) \mathrm{m} / \mathrm{s}$. A hydrogen ion would take a minimum of about $2 \mu$ s to drift to a 
collector $15 \mathrm{~cm}$ away; since the bunches are separated by only about $17 \mathrm{~ns}$, the contributions of multiple bunches will overlap, making a measurement of time of flight impossible.

A measurement of the cutoff of the momentum spectrum might be possible, however. Earlier we estimated that for a residual pressure of $10^{-8}$ torr in the beam tube we would expect to produce about $5 \times 10^{8}$ ions $/ \mathrm{cm}-\mathrm{s}$, of which we might expect to detect of the order of $1 \%$. If we average over all the bunches in the ring, the resolution of the average bunch rms radius will be determined by the resolution of the momentum analyzer used; i.e., $\Delta \sigma / \sigma=-\Delta p / p$, and for $1 \%$ resolution in rms value we need a momentum analyzer with $1 \%$ resolution. The sensitivity to aspect ratio should be small, since this ratio at the secondary focus is only $\sim 1.4$. This effect can be calibrated out using the flying wires, or two orthogonal analyzers can be used.

In the Collider the secondary ions will be subjected to multiple kicks from successive bunches as they exit the beam tube. Under the above conditions a hydrogen atom born with the peak velocity will take about $200 \mathrm{~ns}$ to reach the beam tube wall, during which time it will experience about 12 additional kicks that will increase its momentum by $\sim 25 \%$. The effect of these multiple kicks on ions originally starting with lesser velocities and with different masses must be modelled to determine whether this is a practical diagnostic. Its usage in the lower energy accelerator chain is probably limited because the beam is much larger in radius, the kick size proportionally smaller, and the contribution of kicks from successive bunches much more important. 


\section{REFERENCES}

A1. W. H. DeLuca, "Beam Detection using Residual Gas Ionization," IEEE Trans. Nucl. Sci. NS-16 813-822 (1969).

A2. P. Yu. Komissarov et al., "High-Sensitive Remote Diagnostics of the Accelerated Particles' Beam Cross Section," Proc. 1991 Part. Acc. Conf. (San Francisco), pp. 1166-68.

A3. A. N. Stillman et al., "Mesign of the AGS Booster Ionization Profile Monitor," Proc. 1991 Part. Acc. Conf. (San Francisco), pp. 1189-91.

A4. B. Vosicki and K. Zankel, "The Sodium Curtain Beam Profile Monitor of the ISR," IEEE Trans. Nucl. Sci. NS-22 1475-78 (1975).

A5. R. Galiana et al., "A Carbon Jet Beam Profile Monitor for LEAR," Proc. 1991 Part. Acc. Conf. (San Francisco), pp. 1198-1200.

A6. F. F. Rieke and W. Prepejchal, "Ionization Cross Sections of Gaseous Atoms and Molecules for High-Energy Electrons and Positrons," Phys. Rev. A 6 1507-19 (1972).

A7. F. Hornstra, "Residual Gas Ionization Beam Profile Monitors for the HERA Proton Machines," Proc. 1998 Euro. Part. Accel Conf., 2 1160-62.

A8. K. H. Mess, private communication.

A9. A. G. Chilingarov et al., "The Non-Destructive Ionization LEB Beam Profile Monitor," INP-SSCL Collaboration Proposal, (1992).

A10. Roterto Aiello, private communication.

f.11. J. S. Fraser, "De velopments in Non-Destructive Beam Diagnostics," IEEE Trans. Nucl. Sci., NS-28 2137-41 (1981).

A12. D. D. Chamberlin et al., "Imagescope to Photodiode Beam-Profile Imaging System," IEEE Trans. Nucl. Sci. NS-30 2201-03 (1983).

A13. B. G. Chidley et al., "RFQ1 Diagnostic Devices," Proc. 1990 Lin. Acc. Conf., LANL Report LA-12004-C, 459-61 (March 1991).

A14. A. W. Maschke, "A Nondestructive Method for Beam I'rofile and Absolute Position Measurement," SSC internal memo (June 3, 1992).

A15. R. H. Miller et a!., "Nonintercepting Emittance Monitor." Proc. Xilth Int. Conf. H. E. Accel, (HEACC'83) Fermilab (1983), 602-05.

Ai6. J. C. Sheppard et al., "Ir uplementation of Nonintercepting Energy Spread Monitors," Proc. 1987 Part. Acc. Conf. (Washington).

A17. Y. Yin, "A Multistrip Beam Profile High Order Moment Monitor," Proc. XVth Int. Conf. H. E. Accel, (HEACC'92) Hamburg (July 20-24, 1992).

A18. D. Boussard, "Schottky Noise and Beam Transfer Function Diagnostics," CERN SPS/86-11 (ARF) (1986).

A19. D. A. Goldberg and G. R. Lambertson, "Schottky Monitors for the Tevatron Collider," LBL Report BECON-61/LBID-1129 (1986). 
A20. T. Shintake et al., "Design of Laser-Compton Spot Size Monitor," Proc. XVth Int. Conf. H. E. Accel, (HEACC'92) Hamburg (July 20-24, 1992).

A21. J. Buon, "Possibility to Measure Very Small Spot Sizes Using Gas Ionization at Future Linear Colliders," Part. Accel. 31 39-46 (1990).

A22. J. Buon et al., "The Orsay Spot Size Monitor for the Final Focus Test Beam," Proc. XVth Int. Conf. H. E. Accel, (HEACC'92) Hamburg (July 20-24, 1992). 

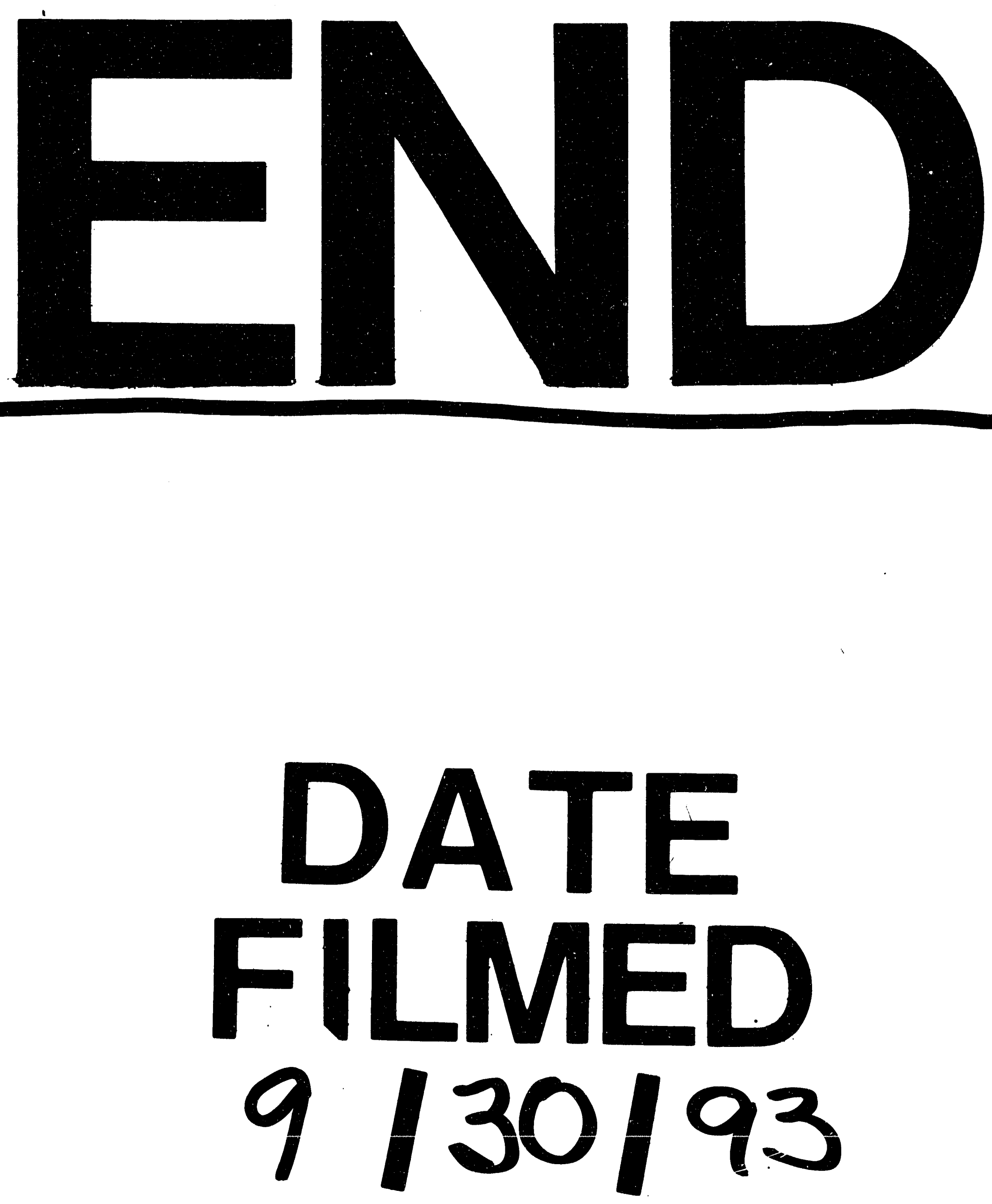
